\title{
Research Paper \\ The Relationship Between Emotion Regulation and Marital Satisfaction Using the Actor-partner Interdependence Model
}

\author{
*Fatemeh Riahi ${ }^{1}$ (), Mahmood Golzari², Fereshteh Mootabi²
}

1. Department of Clinical and General Psychology, Faculty of Psychology and Educational Science, Allameh Tabataba'i University, Tehran, Iran. 2. Family Research Institute, Shahid Beheshti University, Tehran, Iran.

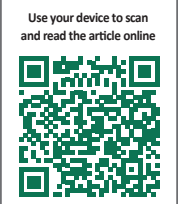

citat on Riahi F, Golzari M, Mootabi F. [The Relationship Between Emotion Regulation and Marital Satisfaction Using the Actor-partner Interdependence Model (Persian)]. Iranian Journal of Psychiatry and Clinical Psychology. 2020; 26(1):44-63. http:// dx.doi.org/10.32598/ijpcp.26.1.3038.1

doi http://dx.doi.org/10.32598/ijpcp.26.1.3038.1

Received: 30 Jan 2019

Accepted: 28 Sep 2019

Available Online: 01 Apr 2020

Key words:

Emotions, Emotion

regulation, Marriage,

Marital satisfaction,

Interpersonal rela-

tions.

\begin{abstract}
A B S T R A C T
Objectives The present study aimed to investigate the relationship between total emotion regulation and the multiple emotion regulation dimensions with marital satisfaction. The study also attempted to assess the moderating role of gender.

Methods A group of 156 married couples located in Tehran were recruited by convenience sampling. Participants rated their emotion regulatory abilities and their marital satisfaction by responding to the Difficulties in Emotion Regulation Scale (DERS) and Dyadic Adjustment Scale (DAS). In order to explore interpersonal effects, data were analyzed using the Actor-Partner Interdependence Model (APIM).

ResultsBoth in men and women, the ability to regulate emotions was predictive of self and partner marital satisfaction. Women's scores in all 6 aspects of emotion regulation and men's scores in all aspects except from "awareness" was positively associated with their own satisfaction. Men's acceptance of emotions, goal-directed behavior, impulse control, and access to regulation strategies predicted their wives' satisfaction. Women's impulse control, access to regulation strategies, and clarity of emotions predicted their husbands' marital satisfaction. The analysis did not support a moderating role for gender.

Conclusion The findings highlight the interconnectedness between partners in the domain of emotions. It seems promising to focus on emotion regulation and its multiple dimensions in the assessment and treatment of couple problems. The use of dyadic methods of analysis is suggested in order to deepen the understanding of relationship phenomena.
\end{abstract}

\section{Extended Abstract}

\section{Introduction}

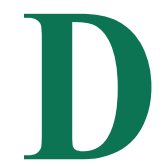

uring recent years, research has paid attention to the role of emotion regulation in interpersonal relationships. According to Gross [4], emotion regulation encompasses "the processes by which individuals influence which emotions they have, when they have them, and how they experience and express these emotions" (p. 275). Emotion regulation often takes place in social con- texts and both influences and is influenced by our relationships with others. Research indicates that individuals who regulate their emotions more effectively, experience more positive social interactions and less conflict with peers [6]. Given the high levels of intimacy between couple partners, emotion regulation processes are expected to play a pivotal role in marital relationships. Since intimacy requires certain degrees of openness and vulnerability, intimate relationships tend to be emotionally challenging. In order to effectively respond to these challenges, partners need to possess a range of skills, including the ability to identify, appropriately express and manage emotions $[7,8]$. Within

\section{* Corresponding Author:}

Fatemeh Riahi, MSc.

Address: Department of Clinical and General Psychology, Faculty of Psychology and Educational Science, Allameh Tabataba'i University, Tehran, Iran. Tel: +98 (912) 8038125

E-mail: f.y.riahi@gmail.com 
the context of intimate relationships, each partner's behaviors, emotional responses, and emotion regulation strategies may act as emotional stimuli for the other partner. Therefore, maintaining an optimal emotional atmosphere in the relationship relies on both partners' regulatory capacities.

The majority of studies in this field have not taken account of the multidimensional nature of emotion regulation. Based on Gratz and Roemer's theory, emotion regulation is not limited to controlling emotional responses but rather entails a set of abilities including: a) awareness of emotions, b) clarity of feelings, c) acceptance of emotional experiences, d) impulse control, e) access to emotion regulation strategies, and f) ability to engage in goal-directed behavior while experiencing negative emotions [11]. Some empirical evidence suggests that the dimensions of emotion regulation may independently contribute to the quality of marital relationships. For instance, in an analysis of couple seeking therapy, men and women's access to emotion regulation strategies was positively associated with their own relationship satisfaction. In addition, women's higher impulse control predicted more satisfaction for their partners. The study failed to find associations between overall emotion regulation and relationship satisfaction. Despite the valuable insights provided, these results were restricted to a relatively small clinical sample and cannot be generalized to community populations [20].

The present research aimed to investigate the relationship between the multidimensional construct of emotion regulation and marital satisfaction in Iranian couples. Considering the scarcity and inconsistency of evidence regarding gender differences in this domain, the study also attempted to as- sess the moderating role of gender in the relationship between emotion regulation and marital satisfaction.

\section{Method}

In light of the interconnected and recursive nature of emotion regulation in the couple context, data were collected and analyzed at a dyadic level. A group of 156 married heterosexual couples located in Tehran were recruited by convenience sampling. Using the Difficulties in Emotion Regulation Scale (DERS), participants rated themselves on 6 aspects of emotion regulation. They also reported their degree of relationship satisfaction by responding to the Dyadic Adjustment Scale (DAS). This study is among the first in Iran to use the Actor Partner Interdependence model (APIM). Actor and partner effects and the effects of gender (as the distinguishing variable) were estimated by Structural Equation Modeling.

\section{Results}

As displayed in Figure 1, both men and women's ability to regulate emotions predicted their own and their partner's relationship satisfaction. The model accounted respectively for $25 \%$ and $20 \%$ of the variation in women and men's marital satisfaction.

Estimated actor and partner effects for each of the emotion regulation dimensions are presented in Table 1. It can be seen that women's scores in all 6 aspects of emotion regulation and men's scores in all aspects except from "awareness" predicted their own relationship satisfaction. Men's acceptance of emotions, goal-directed behavior, im-

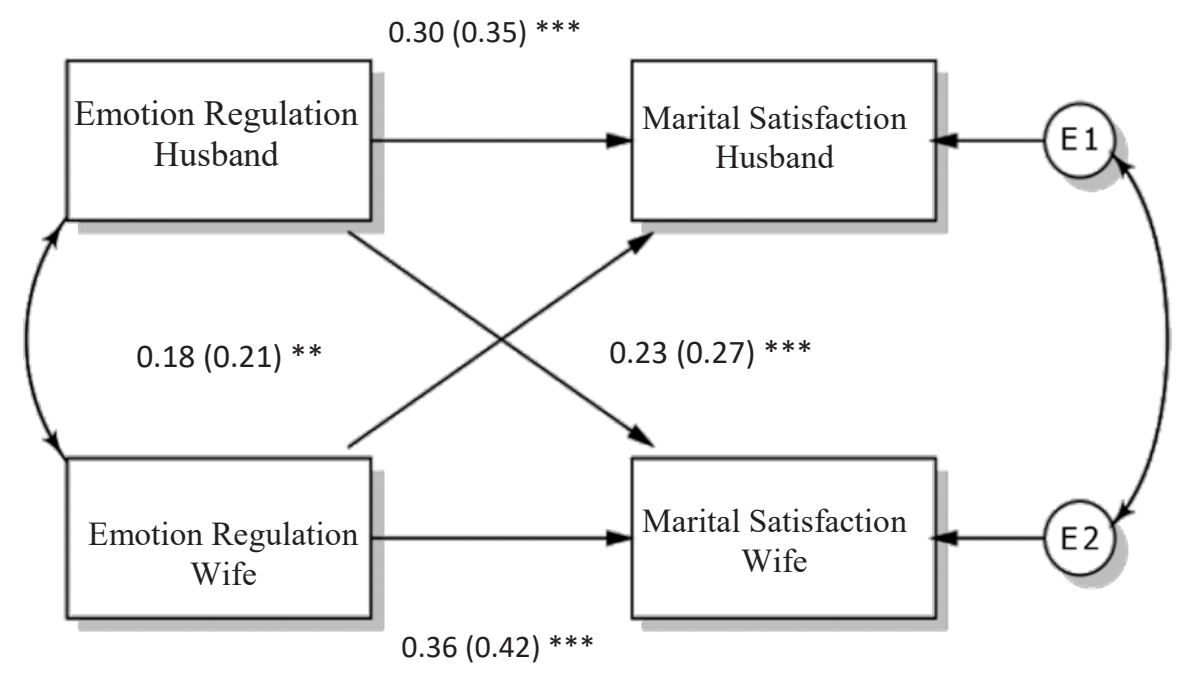

Figure 1. APIM for total emotion regulation

$* \mathrm{P}<0.05 ; * * \mathrm{P}<0.01 ; * * * \mathrm{P}<0.001$ 
Table 1. APIM estimates for dimensions of emotion regulation

\begin{tabular}{|c|c|c|c|c|c|c|}
\hline Dimension & Gender & Effect & Estimate & $95 \% \mathrm{Cl}$ & $\beta$ & $\mathbf{P}$ \\
\hline & & Actor & 1.54 & $0.77-2.31$ & 0.33 & $<0.01$ \\
\hline & Women & & & & & \\
\hline & & Partner & 0.37 & $-0.43-1.71$ & 0.08 & 0.36 \\
\hline \multicolumn{7}{|l|}{ Awareness } \\
\hline & & Actor & 0.61 & $-0.16-3.81$ & 0.13 & 0.09 \\
\hline & Men & & & & & \\
\hline & & Partner & 0.68 & $-0.12-4.71$ & 0.14 & 0.054 \\
\hline & & Actor & 2.28 & $1.44-3.12$ & 0.41 & $<0.001$ \\
\hline & Women & & & & & \\
\hline & & Partner & 0.67 & $0.26-1.6$ & 0.12 & 0.16 \\
\hline \multicolumn{7}{|c|}{ Understanding } \\
\hline & & Actor & 1.16 & $0.2-0.31$ & 0.21 & 0.006 \\
\hline & Men & & & & & \\
\hline & & Partner & 1.32 & $0.39-2.25$ & 0.24 & $<0.001$ \\
\hline & & Actor & 0.92 & $0.34-1.5$ & 0.25 & 0.002 \\
\hline & Women & & & & & \\
\hline & & Partner & 0.77 & $0.14-1.4$ & 0.21 & 0.017 \\
\hline \multicolumn{7}{|l|}{ Acceptance } \\
\hline & & Actor & 0.92 & $0.34-1.5$ & 0.25 & 0.001 \\
\hline & Men & & & & & \\
\hline & & Partner & 0.36 & $0.27-0.99$ & 0.1 & 0.168 \\
\hline & & Actor & 1.39 & $0.87-1.90$ & 0.38 & $<0.001$ \\
\hline & Women & & & & & \\
\hline & & Partner & 1.07 & $0.50-1.64$ & 0.30 & $<0.001$ \\
\hline \multicolumn{7}{|c|}{ Impulse Control } \\
\hline & & Actor & 1.31 & $0.79-1.83$ & 0.36 & $<0.001$ \\
\hline & Men & & & & & \\
\hline & & Partner & 0.82 & $0.25-1.39$ & 0.23 & $<0.001$ \\
\hline & & Actor & 1.35 & $0.51-2.19$ & 0.24 & 0.002 \\
\hline & Women & & & & & \\
\hline & & Partner & 1.73 & $0.82-2.64$ & 0.31 & $<0.001$ \\
\hline \multicolumn{7}{|c|}{ Goal-directed Behavior } \\
\hline & & Actor & 1.71 & $0.87-2.55$ & 0.31 & $<0.001$ \\
\hline & Men & & & & & \\
\hline & & Partner & 0.62 & $0.29-1.53$ & 0.11 & 0.097 \\
\hline & & Actor & 1.17 & $0.74-1.61$ & 0.39 & $<0.001$ \\
\hline & Women & & & & & \\
\hline & & Partner & 0.85 & $0.38-1.32$ & 0.28 & $<0.001$ \\
\hline \multicolumn{7}{|l|}{ Overall } \\
\hline & & Actor & 0.96 & $0.53-1.4$ & 0.32 & $<0.001$ \\
\hline & Men & & & & & \\
\hline & & Partner & 0.65 & $0.18-1.12$ & 0.23 & 0.001 \\
\hline
\end{tabular}


pulse control, and access to emotion regulation strategies was positively associated with their wives' satisfaction. Women's impulse control, access to regulation strategies, and clarity of emotions was predictive of their husbands' relationship satisfaction.

The test of the interactions of gender with the actor effect was not statistically significant, chi square $(1)=1.518$ $(\mathrm{p}=.218)$, and the test of the interactions of gender with the partner effect was also not statistically significant, chi square $(1)=0.007(p=.932)$. The combined test of the interactions of gender with both the actor and partner effects was not significant either, chi square $(2)=3.668(\mathrm{p}=.160)$. Therefore, the analyses did not support a moderating role for gender.

\section{Discussion}

Findings supported the hypothesis that emotion regulation is positively associated with self and partner marital satisfaction. The results are in line with previous research highlighting the effects of emotion regulation on the quality of relationships [10, 20, 40]. While Bloch et al. and Rick et al. found partner effects of emotion regulation only for women $[10,20]$, in the present study significant partner effects were traced for both women and men. Hence the results emphasize the interconnectedness between partners in the domain of emotions. Since gender did not moderate the relationships between emotion regulation and marital satisfaction, men and women's emotion regulation abilities appear to be to the same extent predictive of their own and their partner's satisfaction.

Based on their clinical observations, Levenson et al. state that although couples seeking professional help present different issues, almost all of them struggle with some kind of problem with the regulation of positive or negative emotions [9]. Many approaches to psychotherapy, including mindfulness and acceptance-based therapies and emotion focused therapy, focus on problems with emotions and emotion regulation $[13,50,51]$. The present study found significant relationships between dimensions of emotion regulation and marital satisfaction, pointing to the importance of understanding emotion regulation as a multidimensional construct. In the assessment of couple problems, paying attention to the different dimensions of emotion regulation could help therapists determine specific intervention targets.

\section{Ethical Considerations \\ Compliance with ethical guidelines}

The participants were informed about the aims and guidelines of the study. Questionnaires were coded and placed in individual envelopes in order to ensure the anonymity and confidentiality of obtained data. All subjects signed a written informed consent form.

\section{Funding}

This study did not receive any specific grant from funding agencies of the public or nonprofit sectors.

\section{Authors contributions}

Conceptualization, validation, review and editing: all authors; Methodology, data analysis, resources, writing: Fatemeh Riahi; Supervision: Mahmoud Golzari, Fereshteh Mootabi.

\section{Conflicts of interest}

The authors declare no conflict of interest.

\section{Acknowledgements}

We are grateful to all the participants involved in this research. 
This Page Intentionally Left Blank 


\title{
رابطه ابعاد تنظيم هيجان و رضايت زناشويى با استفاده از مدل وابستكَى متقابل عامل ـشريك
}

\author{
"فاطمه رياحى' (0، محمود كلزارى'، فرشته موتابى'
}

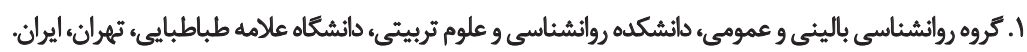

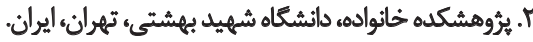

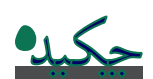

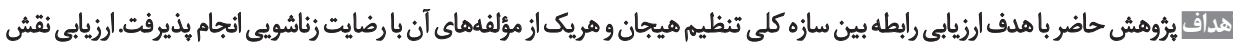

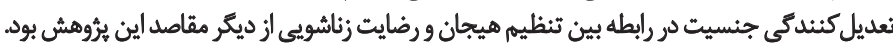

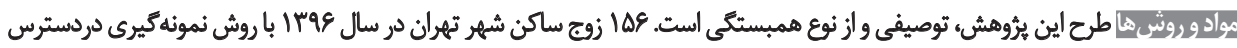

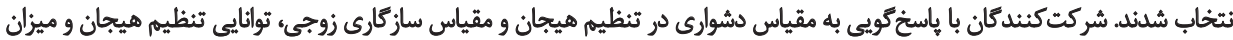

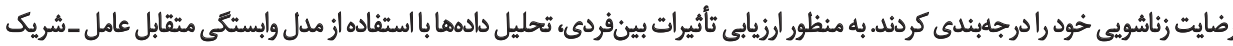

صورت كرفت.

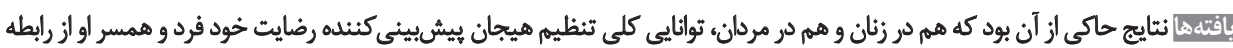

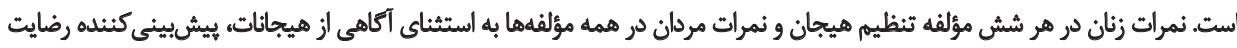

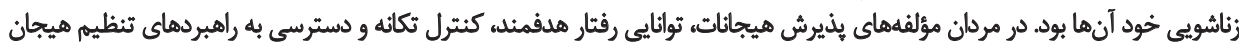

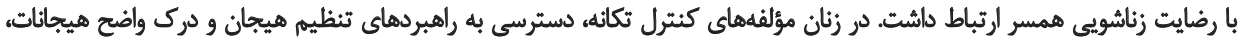

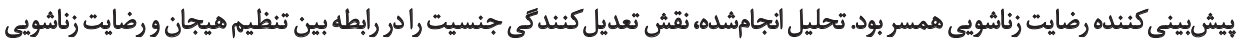
تأييدنكرد.

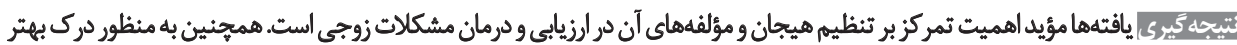

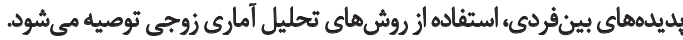

تاريخ دريافت: •ا يهمن Irav

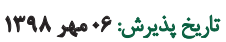

تاريخ انتشار: با فروردين

كليدوازڤها:

هيجان، تنئيم هيجان.

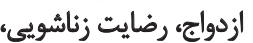

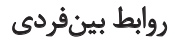

خانواده اصلى و وضعيت اجتماعى ـاقتصادى) و بينفردى (مثلاً

مقدمه

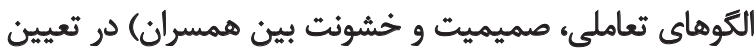

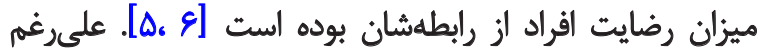

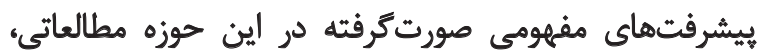

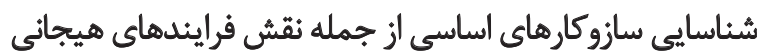

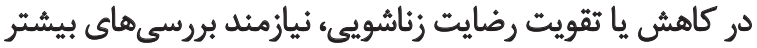

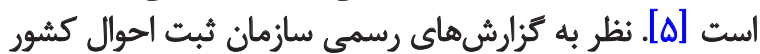

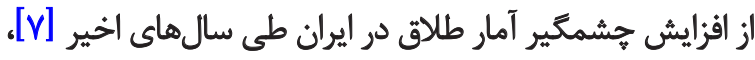

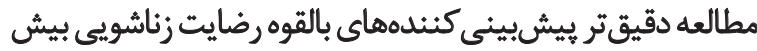

$$
\text { از ييش ضرورى به نظر ميرسد. }
$$

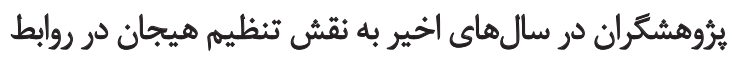

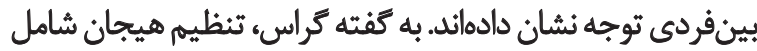

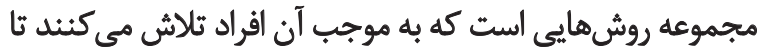

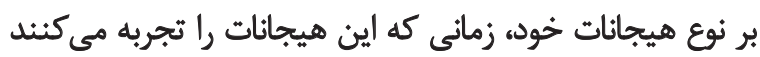

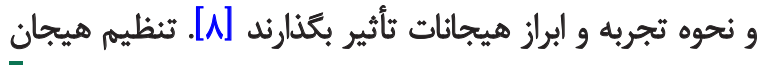

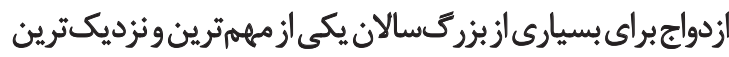

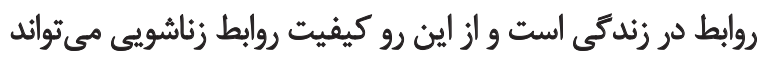

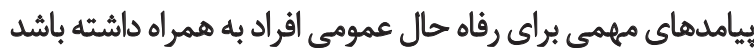

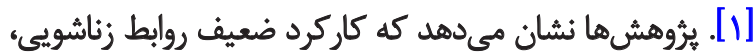

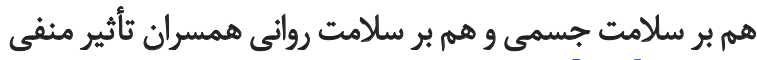

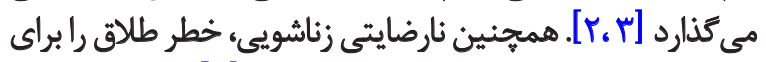

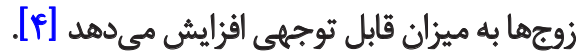
با توجه به مزايايى كه شكل

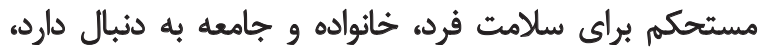

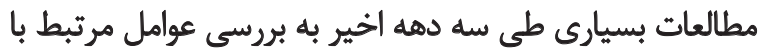

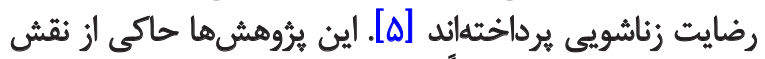

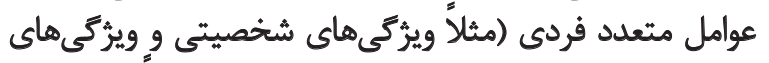
جمعيتشناختى همسران)، زمينهاى و محيطى (مثلأويزّكى هاى

* : نويسئده مسئول:

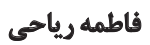
نشائى: تهران، دانشعاه علامه طباطبايى، دانشكله روانشناسي و علوم تربيتى، كروه روانشناسى باليني و عمومى.

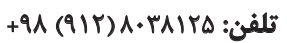
f.y.riahi@gmail.com يست الكترونيكي 


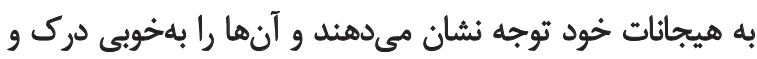

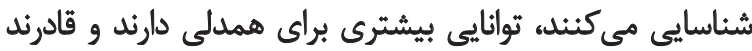

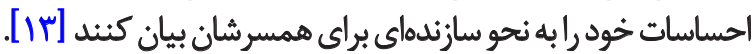
مؤلفه بذيرش هيجانات شامل باز بودن فرد نسبت به تجارب

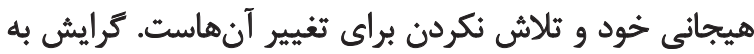

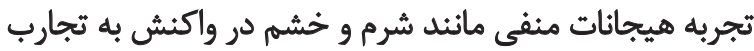

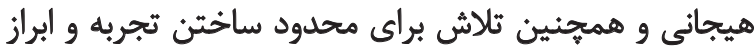

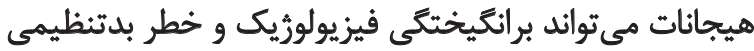

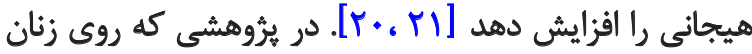

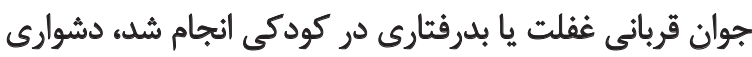

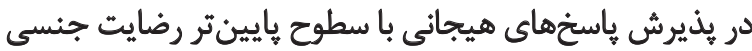

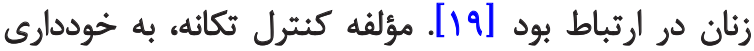

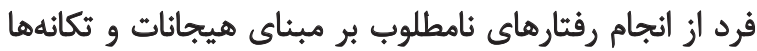

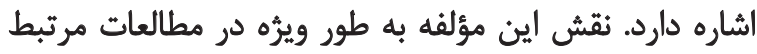

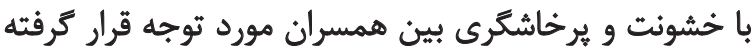

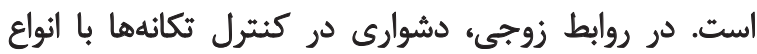

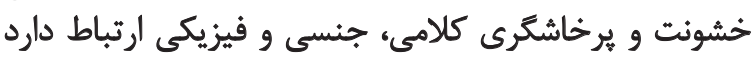

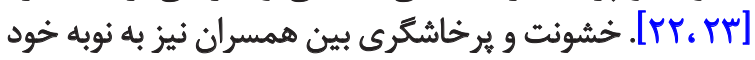

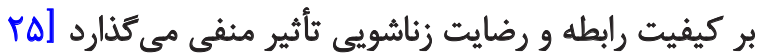

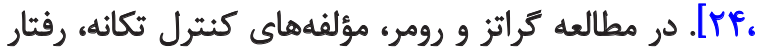

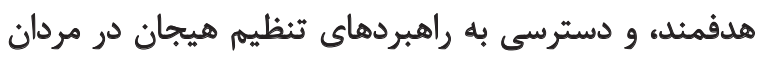
با خشونت و بدرفتارى نسبت به همسر در ارتباط بود [1/]. راهبردهايى كه همسران براى ثنظيم هيجانات خود استفاده

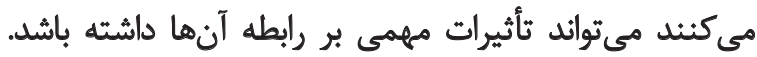

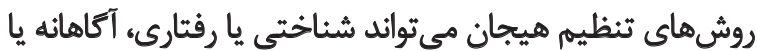

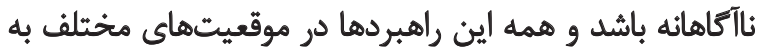

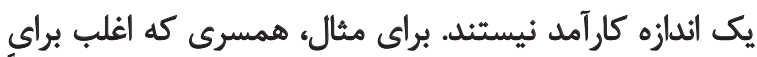

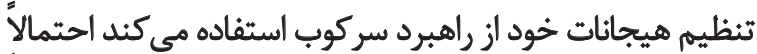

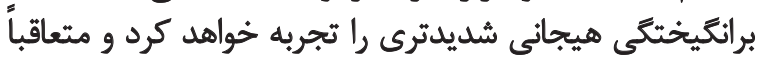

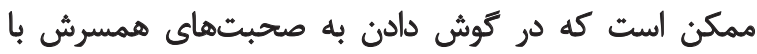

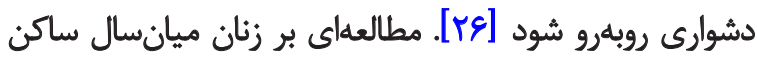

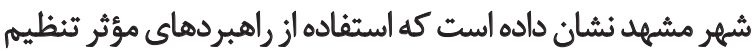

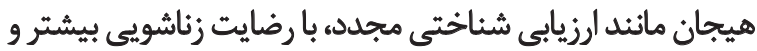

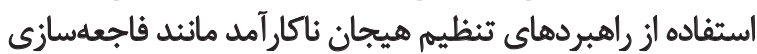

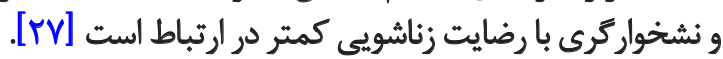

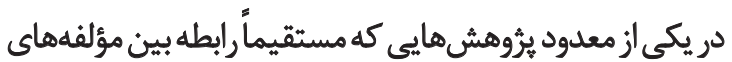

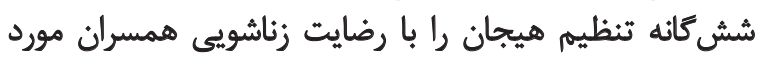

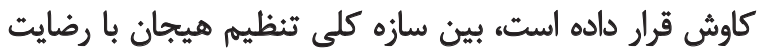

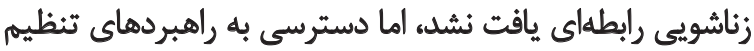

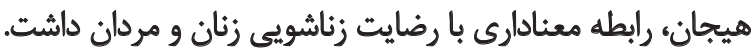

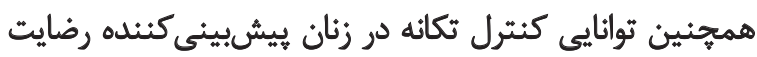

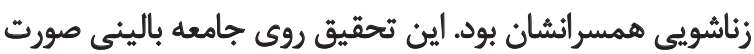

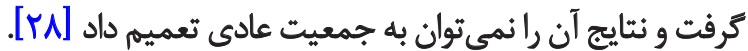

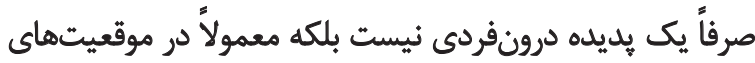

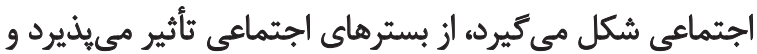

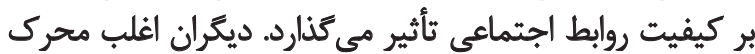

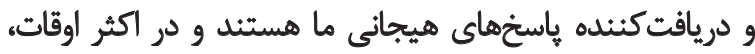

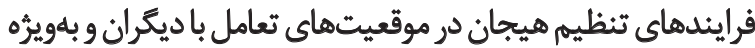

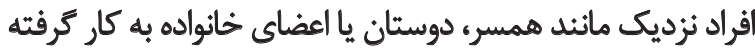

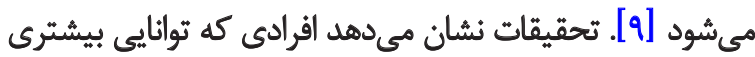

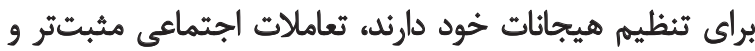

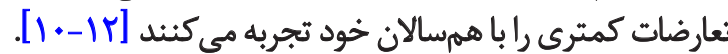
با توجه به سطوح بالاى صميميت بين همسران، به نظر ميرسد

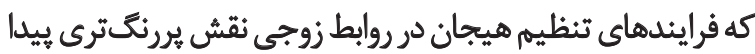

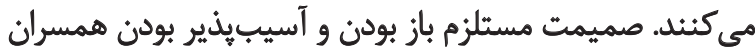

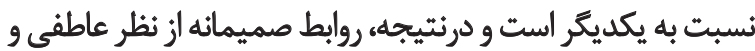

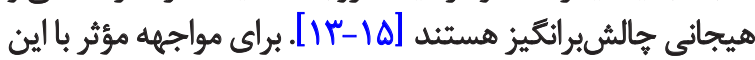

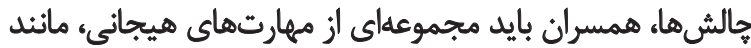

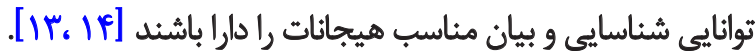

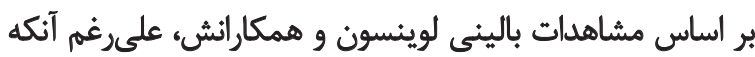

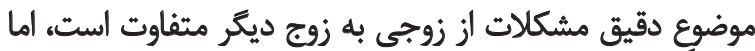

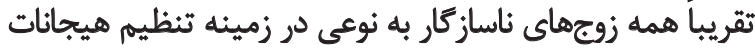

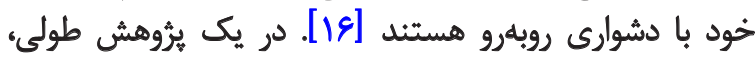

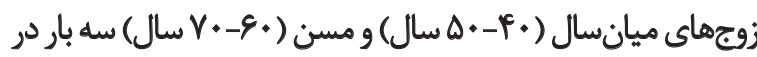

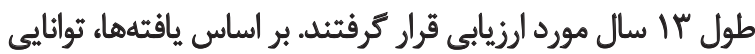

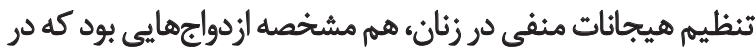

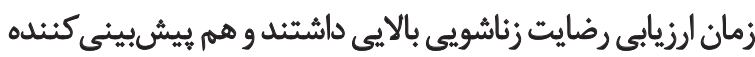

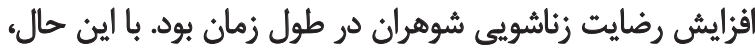

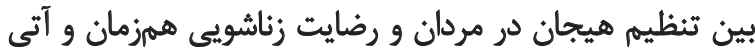

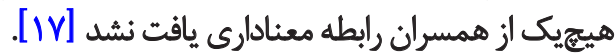

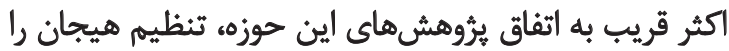

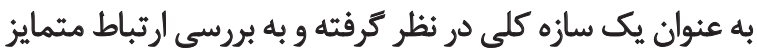

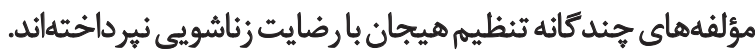

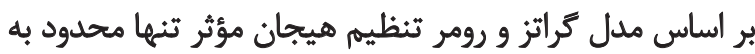

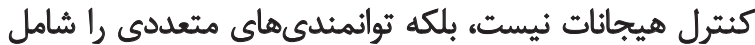

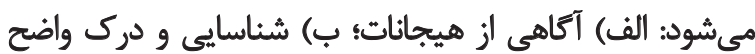

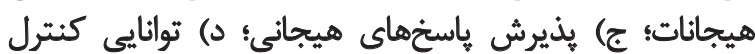

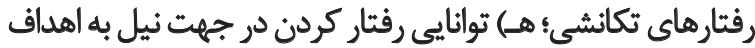

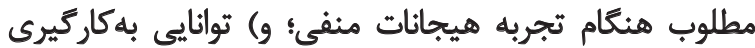

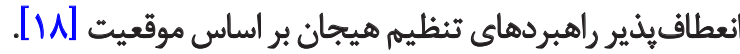
هرخى شواهد تجربى حاكى از آن است كه ابعاد مختلف تنظيميم

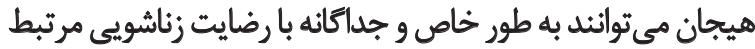

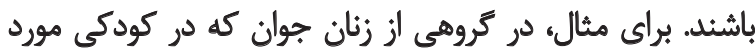

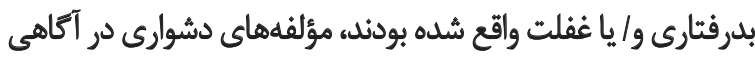

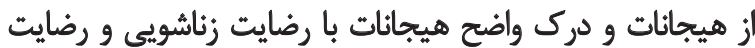

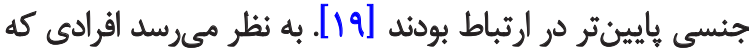




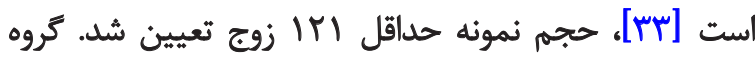

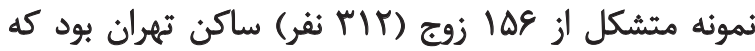

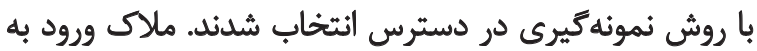

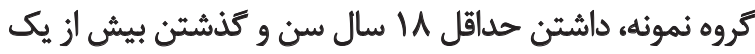

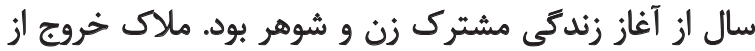

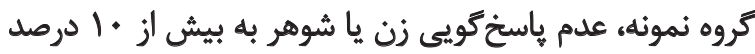

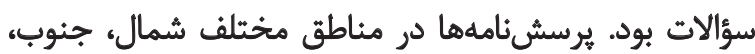

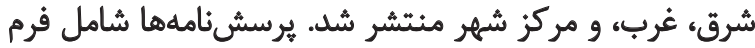

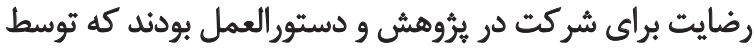

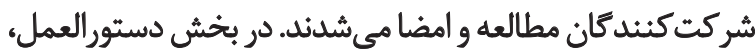

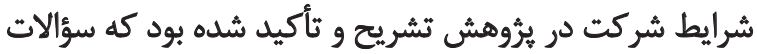

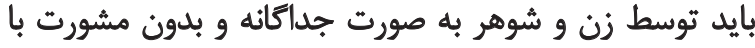

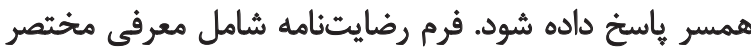

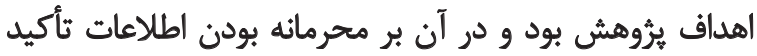

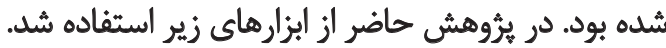

$$
\text { مقياس سازتًارى زوجى }
$$

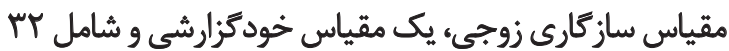

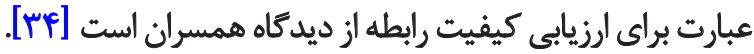

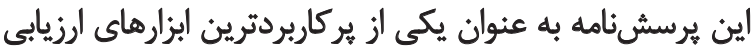

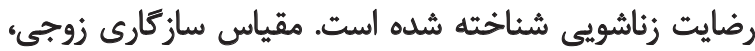

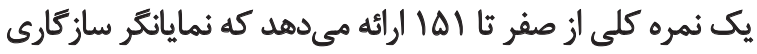

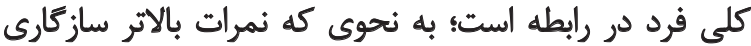

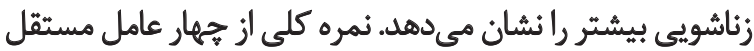

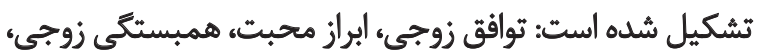

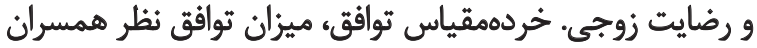

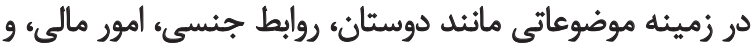

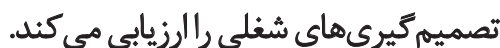

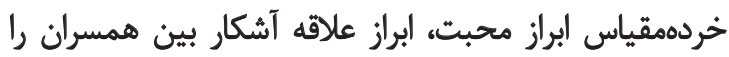

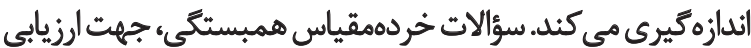

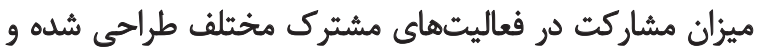

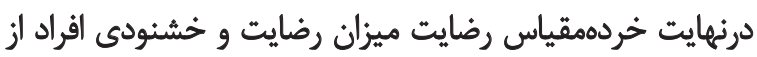

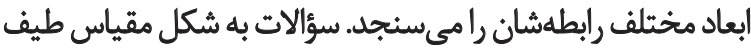

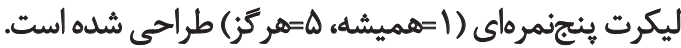

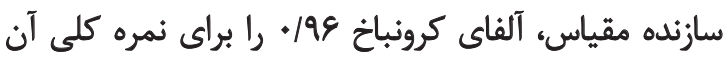

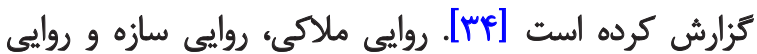

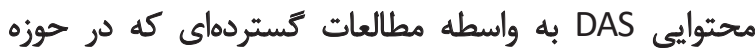

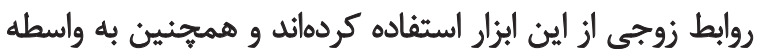

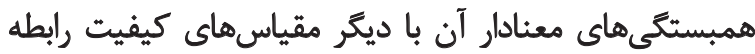

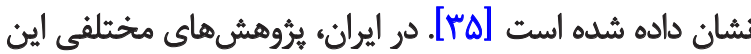

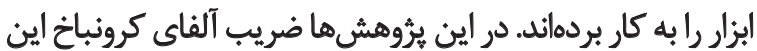

1. Dyadic Adjustment Scale (DAS)

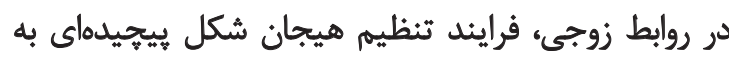

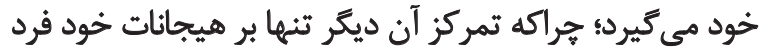

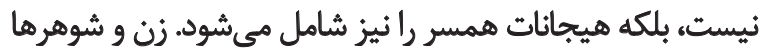

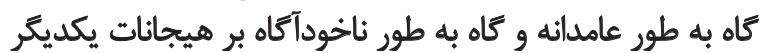

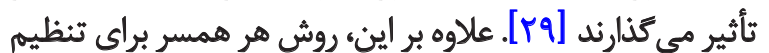

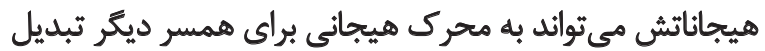

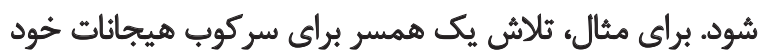

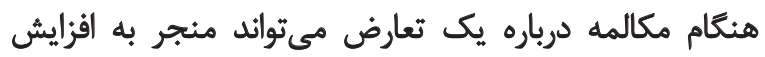

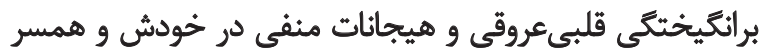

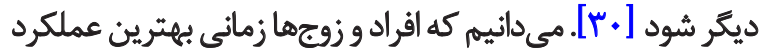

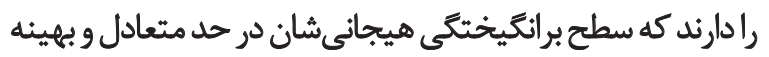

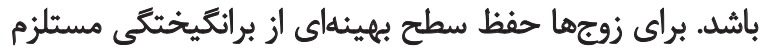

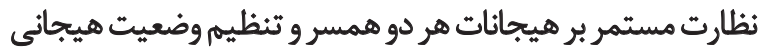

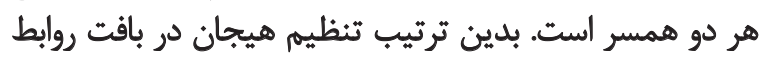

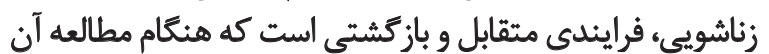

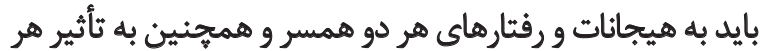

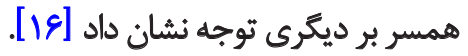

با توجه به شواهد موجود، انتظار محرود كه تنظيم هيجان

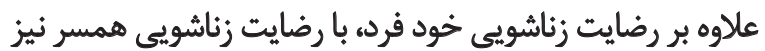

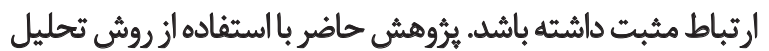

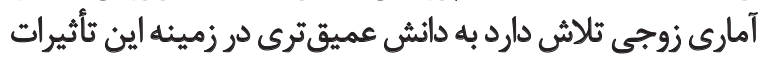

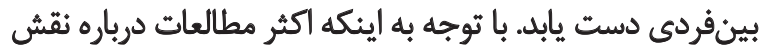

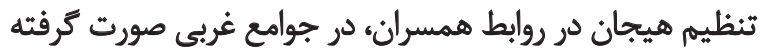

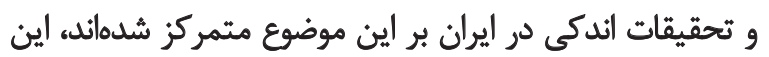

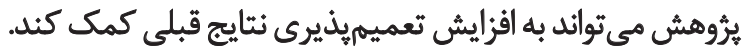
يروهش حاضر، از بررسى تنظيم هيجان به صورت كلى فراتر

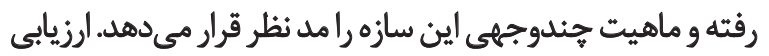

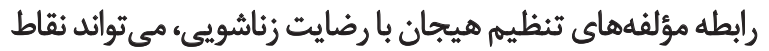

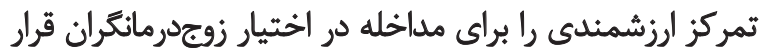

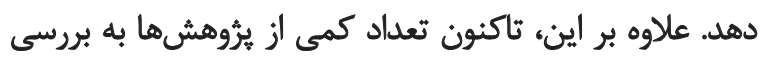

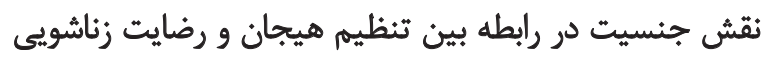

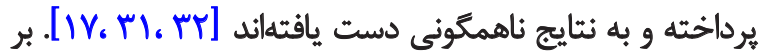

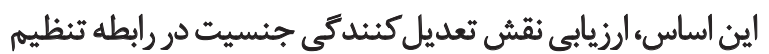

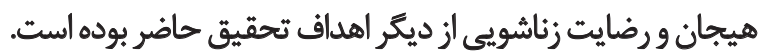

روش

اين بثروهش، توصيفى و از نوع همبستخى است. جامعه آمارى

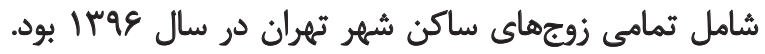

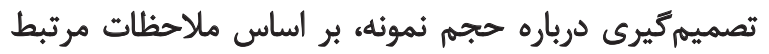

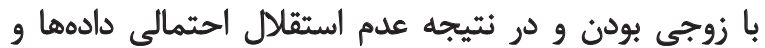

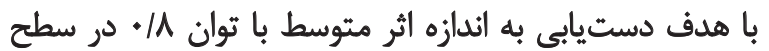

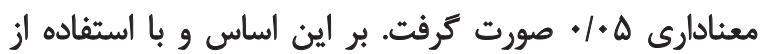

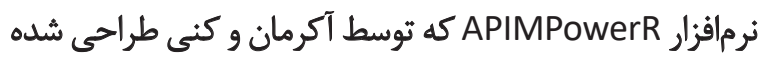




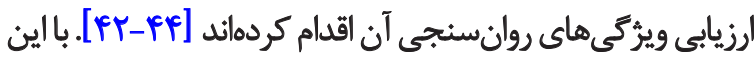

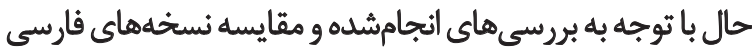

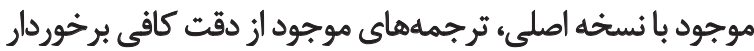

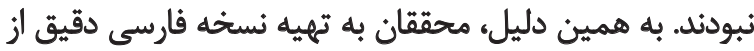

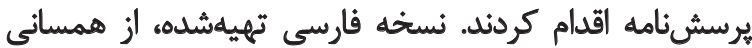

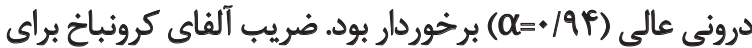

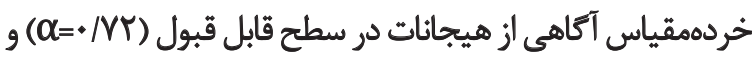

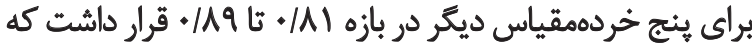

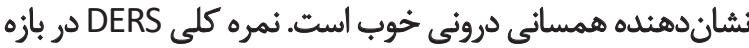

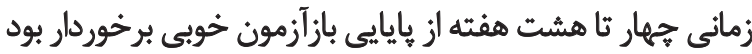

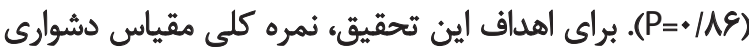

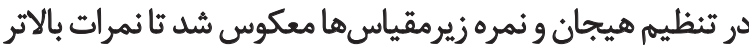
نشاندهنده توانايي بيشتر در تنظيم هيجائات باشديا

تحليل اوليه

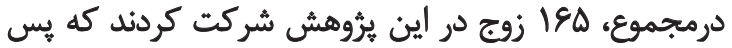

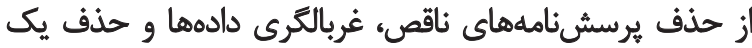

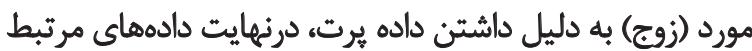
با وها زوج وارد تحليل شد. اطلاعات توصيفى موردنظر از إن جمله

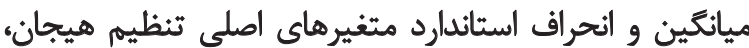

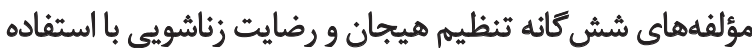

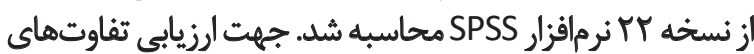

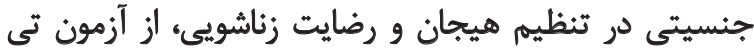

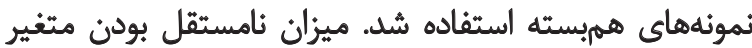

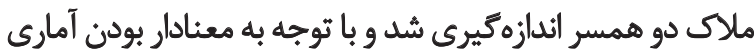

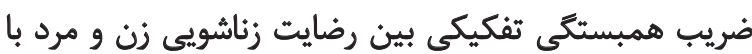

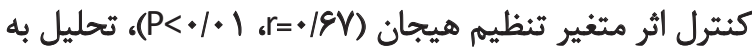
روش زوجى ادامه يافت.

\section{تحليل مدل وابستكّى مثقابل عامل -شريكى}

به منظور مطالعه تأثيرات متقابل ويزئى هاى همسران، دادههاى

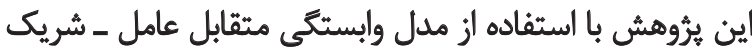

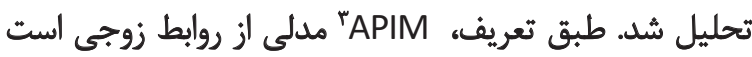

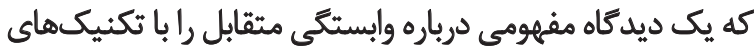

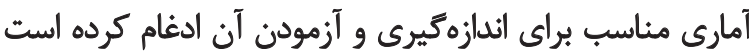

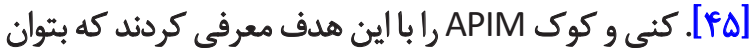

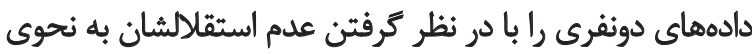

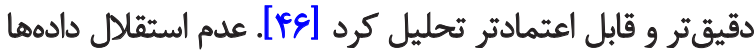

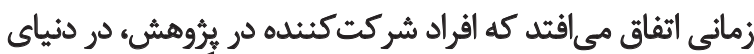

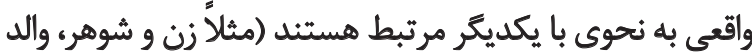
و فرزند، كارفرما و كارمند، دو همشير و غيره) و در نتيجه هئه احتمال

3. Actor-Partner Interdependence Model مدل وابستكي مثيقابل عامل

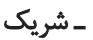

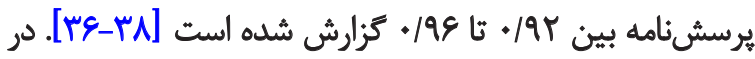

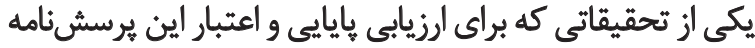

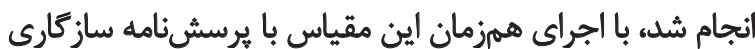

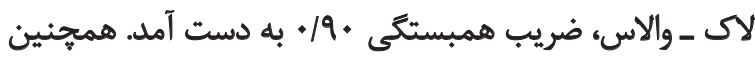

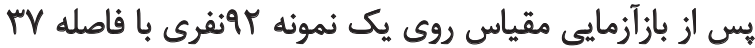

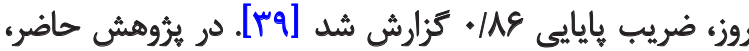

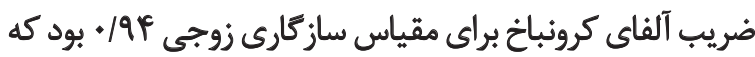

$$
\begin{aligned}
& \text { نشان دهئده همسانى درونى عالى است } \\
& \text { مقياس دشوارى در تنظيم هيجان' }
\end{aligned}
$$

مقياس دشوارى در تنظيم هيجان يك ابزار خودكزارشى و

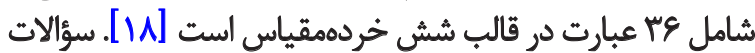

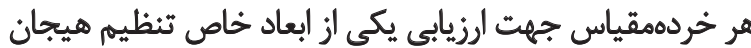

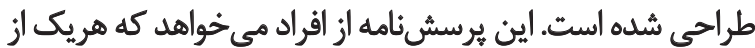

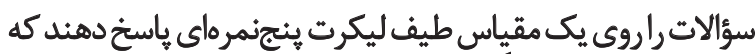

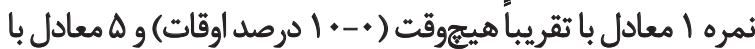

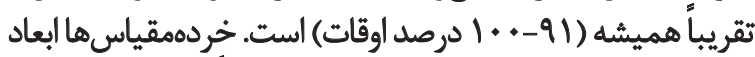

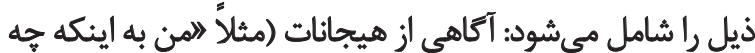

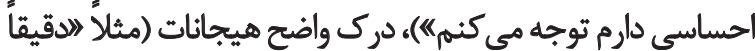

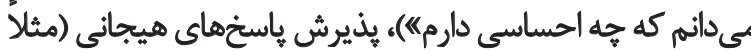

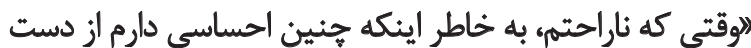

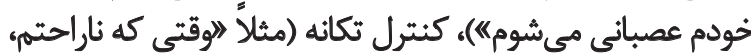

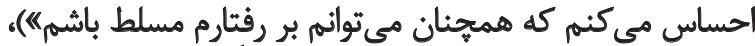

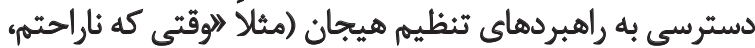

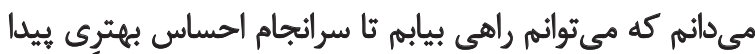

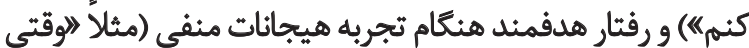

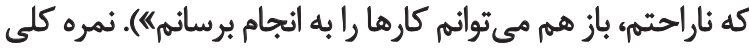

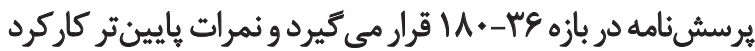
بهتر در تنظيم هيجان را نشان مي دهد.

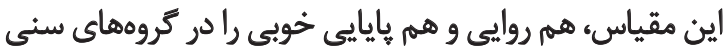

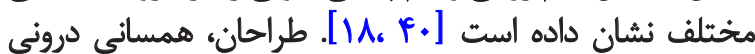

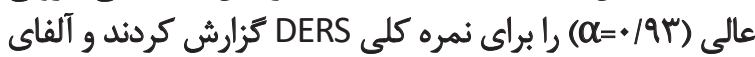

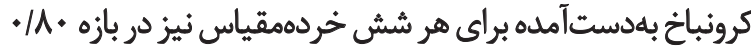

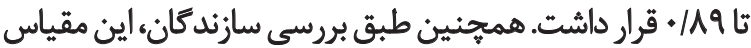

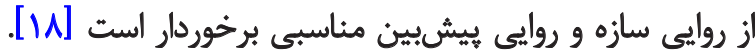

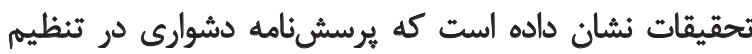

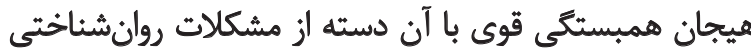

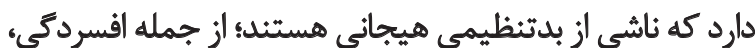

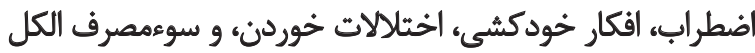

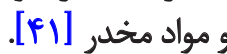
در ايران بُروهشهاى مخثلفى از اين يرسشنامه استفاده و به 


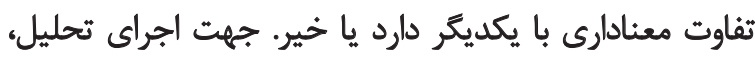

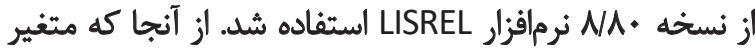

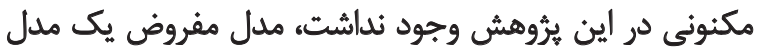

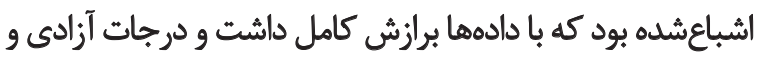
آماره خي دو معادل صفر بود.

1.diต̣!

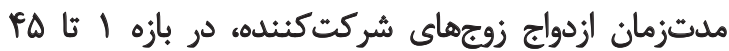

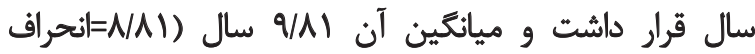

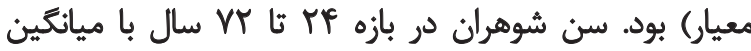


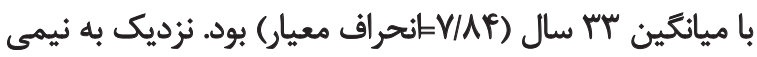

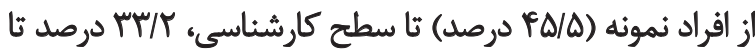

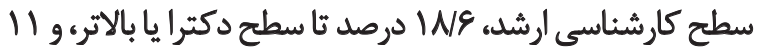

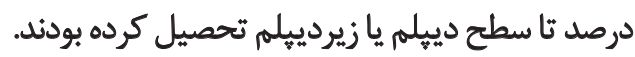

\section{توصيف متغير هاى يُورهش}

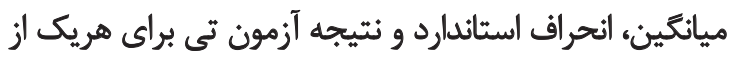

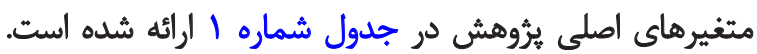

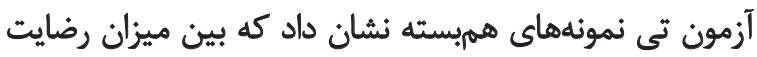

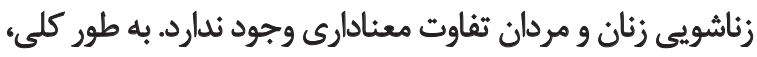

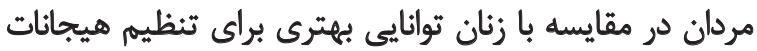

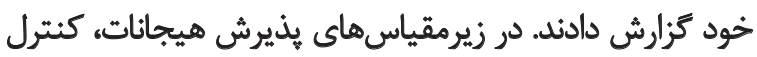

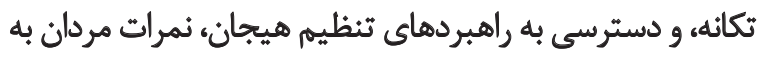

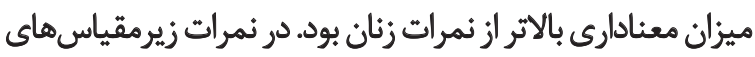

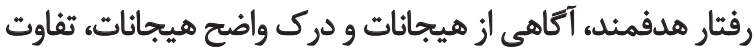
معنادارى بين زنان و مردان ديده نشد.
اينكه بر يكديكر تأثير بكذارند و ياسخهايشان از قبل با يكديكر همبسته باشد زياد است.

در راهبردهاي آمارى سنتى كه فردمحور و بر مبناي روش هادئي

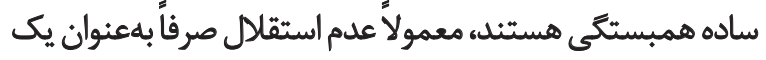

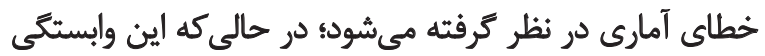

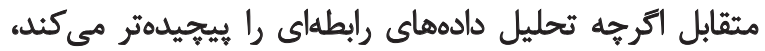

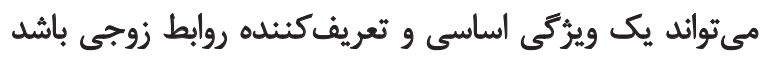

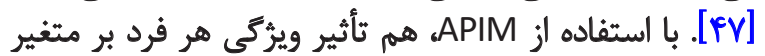

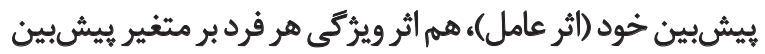

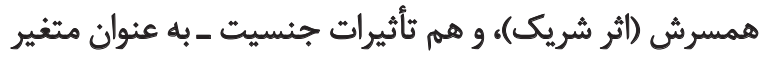

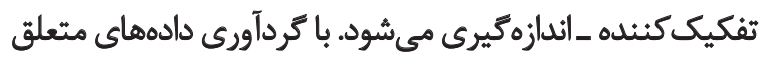

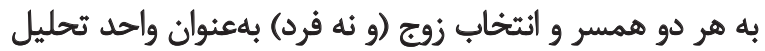

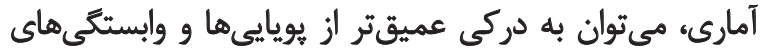

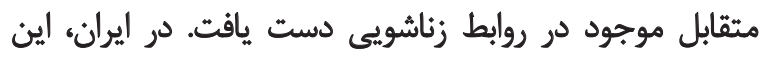

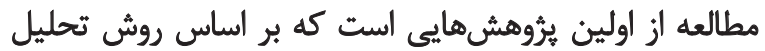
آمارى زوجى مطابق با APIM اجرا شده است.

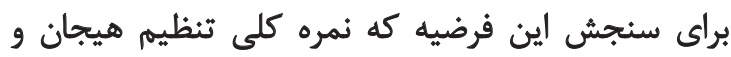

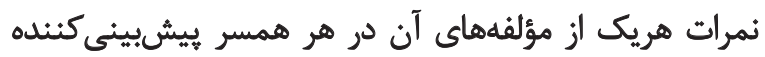

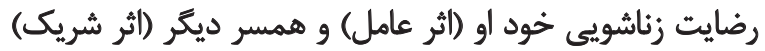

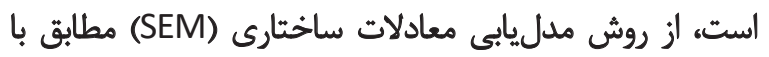

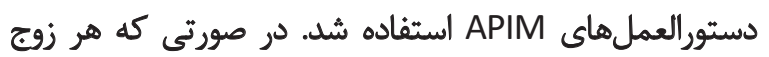

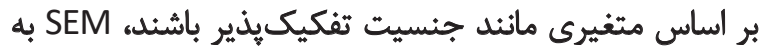

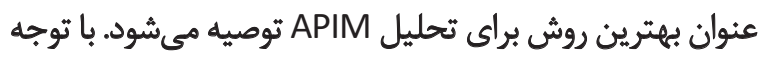

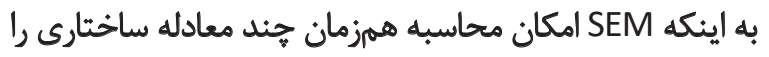

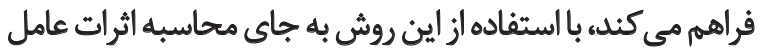

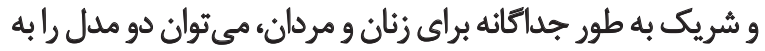

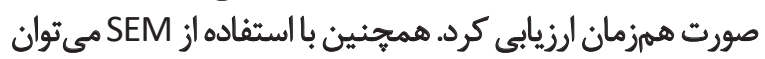

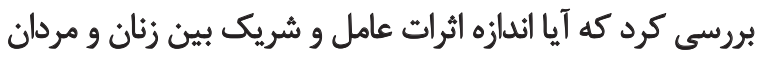

جدول ا. شاخصهاى توصيفى و تفاوت ميانكينهاى زنان و مردان

\begin{tabular}{|c|c|c|c|c|c|c|}
\hline سطح معنادارى & $\mathrm{t}$ & تفاوت ميانكينها & بازه مقياس & مر دان ثيانحراتئن معيار & ميانكين زنان بانحرأف & مثغير \\
\hline $.1 \cdot \pi$ & $-Y / Y A$ & $-\Delta / 4 A$ & M. & $9 q / \Lambda \cdot \pm r \cdot / T V$ & QR/TYIWWTr & تنظيم هيبجان كلى \\
\hline.$/ \cdot \pi r$ & $-r / 1 \&$ & $-1 / r$ & $r \cdot-q$ & $|W r \Delta \pm F|$ & $\mid V / \backslash \Delta \pm \Delta / 1$ & يأذيرش هيجانات \\
\hline.$/ p q$ & --189 &.$- /$ Me & $r \Delta-\Delta$ & $1 \cdot / N \pm r / M r$ & $1 . / P \vee \pm r / r q$ & رقفتًار هدفمند \\
\hline $1+00$ & -r/AY & $-1 / 8$ & $r+-\varepsilon$ & IE/AY $\pm F / M$ & $\mid \Delta / \pi T \pm \Delta / r \Delta$ & كنترل تكائه \\
\hline.$/ 1 F$ & $1 / 4 A$ & . IAT & $r+-\varepsilon$ & $\mid Q / / A \pm T / N E$ & $\mid Q / A Y \pm T / M$ & أكاهى از هيجانات \\
\hline I.TA & $-r / r a$ & $-1 / \Delta r$ & $f+-1$ & $r / / Y q \pm \Delta / Y q$ & $r+/ r q \pm E / 19$ & راهبردهاى تنظيم هيجان \\
\hline$\cdot / \pi V$ & $-1 / 11$ &.$- / 4$ & $r \Delta-\Delta$ & $\mid Q / g T \pm \Psi / \cdot \Delta$ & $1 Q / T f \pm T / \Psi \wedge$ & درك واضح هيجانات \\
\hline.$/ \cdot V$ & - V/AF & $-1 / 9 Y$ & $|0|-$ & $\mid 1 r / 9 r \pm 19 / 9 \Delta$ & $111 / q \pm \pm 19 / \% \Delta$ & رضايت زناشويىى \\
\hline
\end{tabular}


جدول Y. نتايج تحليل APIM براى نمره كلى تنظيم هيجان و رضايت زناشوييى

\begin{tabular}{|c|c|c|c|c|c|}
\hline سطح معنادارى & ب & فاصله الطمينان 9 درصد & برأورد & اثر & جنسيت \\
\hline$<+|+\infty|$ &.$/ 4 r$ & $\cdot / \pi F-+/ F A$ &.$/ 48$ & عامل & \multirow[b]{2}{*}{ زن } \\
\hline$<\cdot 1 . .1$ &.$/ T V$ & $\cdot N \cdot-\cdot / M$ & $\cdot / \pi T$ & شريك & \\
\hline$<.1 . .1$ & . & $\cdot M A-\cdot / P$ &.$/ \mu$ & عامل & \multirow[b]{2}{*}{ مرد } \\
\hline $.1 . .1$ &.$M I$ & $\cdot N-\cdot / M A$ &.$/ M A$ & شريى & \\
\hline
\end{tabular}

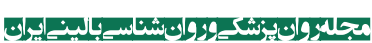

رضايت زناشويى همسر بودند. توانايى كنترل تكانه، دسترسي

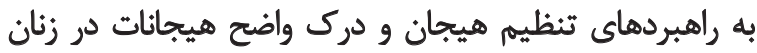

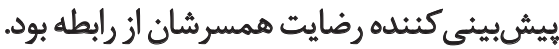

در تحليل مدل مفروض با استفاده از روش SEM، ضرايب

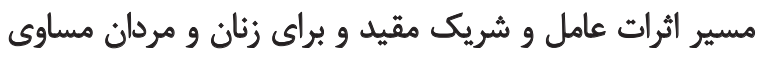

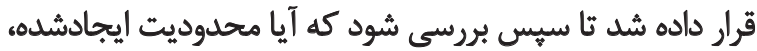

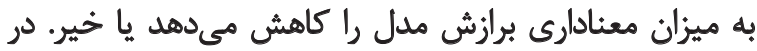

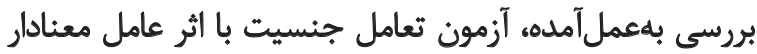
نبود، خيى برو (1)

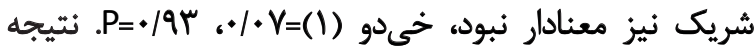
آزمون تركيبى ثعامل جنسيت با هر دو اثر عامل و شريك نيز نيز

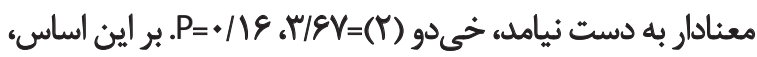
نتايج يُروهش حاضر حاكي از آن است كه جنسيت ديث در رابطه بين تنظيم هيجان و رضايت زناشويي نقش تعديل كنيند إني ندارد.

ثب

يثوهش حاضر با هدف ارزيابى رابطه تنظيم هيجان كلى

\section{ثنايج تحليل مدل وابسيّكي مثقابل عامل ـشريك}

نتايج تحليل APIM با نمره كلي تنظيم هيجان به عنوان متغير

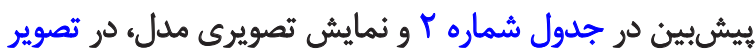

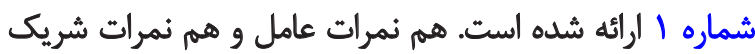

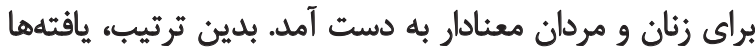

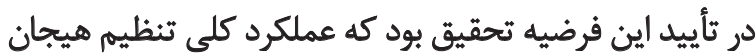

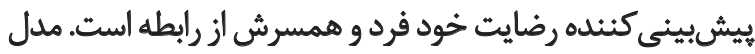

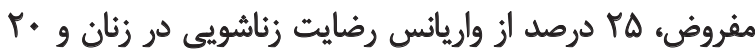

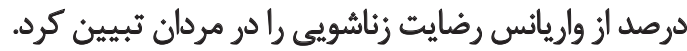
نتايج تحليل APIM با نمره هريك از مؤلفههاى تنظيم هيجان

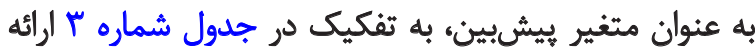

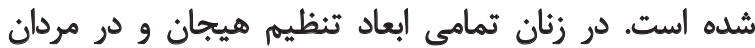

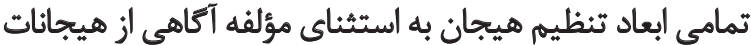

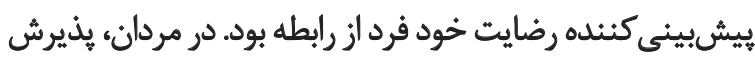

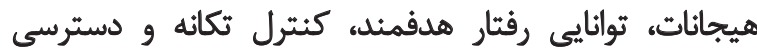

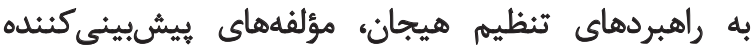

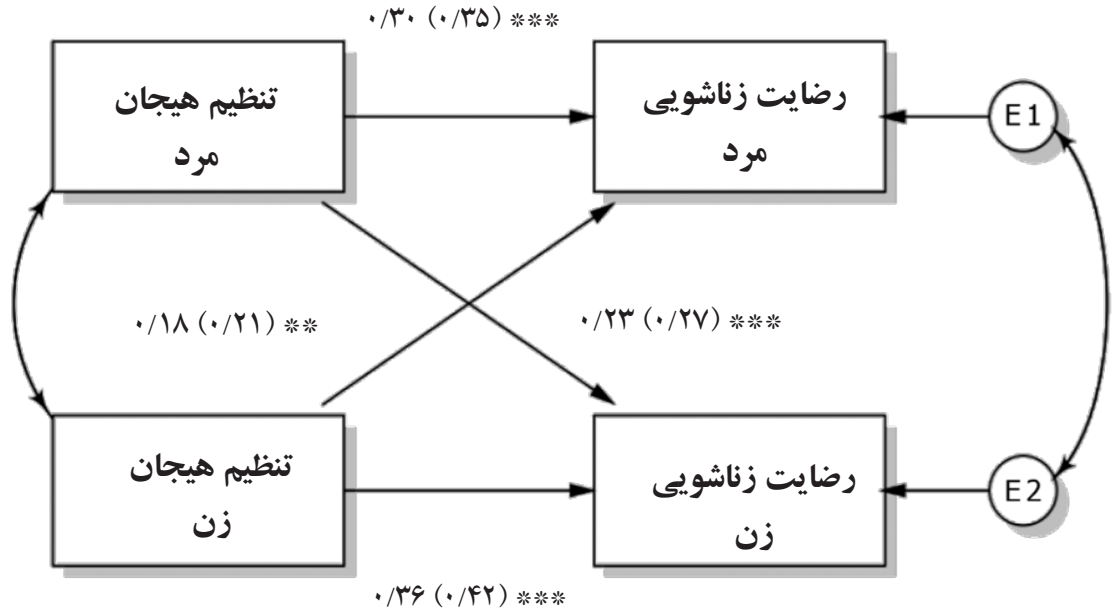


جدول ب. نتايج تحليل APIM براى مؤلفههاي تنظيم هيجان و رضايت زناشويي

\begin{tabular}{|c|c|c|c|c|c|c|}
\hline سطح معنادارى & بتا & فاصله اطمينان هوصد & برأورد & ائي & جنسيت & مؤلفه تنظيم هيجان \\
\hline$<.1 .1$ & $\cdot / \pi$ &. $\mid W-Y / M I$ & $V / \Delta f^{f}$ & عامل & \multirow[b]{2}{*}{ زن } & \multirow{4}{*}{ أكاهى از هيجانات } \\
\hline e &.$/ * 1$ & $-. / 4 T-|M|$ &.$/ M V$ & شريك & & \\
\hline .19 & 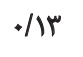 &.$-|1 \varepsilon-r / A|$ &.$|8|$ & عامل & \multirow[b]{2}{*}{ 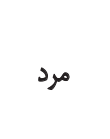 } & \\
\hline 1. $\Delta P$ &.$/ N F$ & $-* / N r-P / M$ &.$/ 8 A$ & شريك & & \\
\hline$<+1++1$ & $+/ 41$ & $M R T-r / M r$ & r/TA & 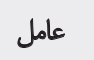 & \multirow[b]{2}{*}{ زن } & \multirow{3}{*}{ درك واضح هيجائات } \\
\hline.$/ 18$ & $+/ T$ & $. / 4 \varepsilon-V / q$ & $.19 V$ & شريك & & \\
\hline $\begin{array}{l}. .9 \\
<\bullet 1 .+1\end{array}$ & $\cdot / M I$ & $\begin{array}{l}. / r-. / M I \\
. / 4 q-r / r \Delta\end{array}$ & $\begin{array}{l}\text { WV } \\
\text { W/MT }\end{array}$ & شريك & مرد & \\
\hline 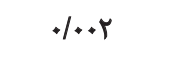 &.$/ T \Delta$ & $. / M \varphi-1 / \Delta$ &.$/ 94$ & عامل & \multirow[b]{2}{*}{ ن } & \multirow{4}{*}{ 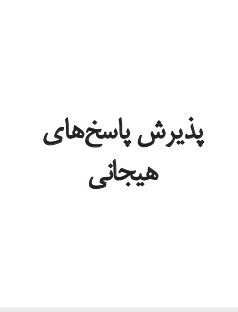 } \\
\hline.$/ \cdot 18$ & $+/ T I$ & $. / 1 f-1 / 4$ & $\cdot M$ & شريك & & \\
\hline$+1+.1$ &.$/ T \Delta$ & $\cdot / m-1 / \Delta$ &.$/ 94$ & عامل & \multirow[b]{2}{*}{ هرد } & \\
\hline.$/ 18 A$ &.$/ 1$ & $. / M V=. / 99$ &.$/ 48$ & شريك & & \\
\hline$<\cdot 1 \cdot \cdot 1$ &.$/ r A$ & $\cdot(A Y-1 / 9$ & $1 / \% 9$ & عامل & \multirow[b]{2}{*}{ زن } & \multirow{4}{*}{ كتترل تكانه } \\
\hline$<+1+\infty 1$ &.$/ \%$ & $\cdot 10+-1 / 4$ & $V / r Y$ & شريك & & \\
\hline$<+1+\cdot 1$ &.$/ 48$ & . $/ M 9-1 / A r$ & $\mid /{ }^{\prime \prime}$ & 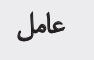 & \multirow[b]{2}{*}{ هرد } & \\
\hline$<+1++1$ & 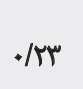 & $. / 1 \% \Delta-1 / 4 q$ & . & شريك & & \\
\hline$+\%+r$ & $+/ K F$ & $\cdot(\Delta)-r / 19$ & $1 / r \Delta$ & 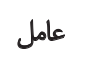 & \multirow[b]{2}{*}{ زن } & \multirow{4}{*}{ رفتار هلفومند } \\
\hline$<\cdot 1 \cdot .1$ &.$/ M$ & . $/ A T-$ T/AF & $M$ & شريك & & \\
\hline$<+1 .+1$ & $\cdot / M$ & $\cdot / A Y-Y / \Delta \Delta$ & $M$ & عامل & \multirow[b]{2}{*}{ هرد } & \\
\hline .1 .97 &.$M$ & $. / r q-1 / \Delta r$ & . $/ 8 T$ & شريك & & \\
\hline$<+1 \cdot+1$ &.$/ 7 q$ & $\cdot\left|M^{2}-\right||F|$ & $\mathrm{V} / \mathrm{V}$ & 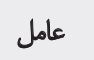 & \multirow[b]{2}{*}{ زن } & \multirow{4}{*}{ دستربسي به راهبردهاي } \\
\hline$<+1++1$ & $\cdot / T A$ & $. / \mu \Lambda-1 / \pi r$ & $\cdot / 1 \Delta$ & شريك & & \\
\hline$<+1 .+1$ & $\cdot / T r$ & $\cdot / \Delta r-1 / F$ &.$/ 98$ & 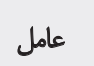 & \multirow[b]{2}{*}{ مرد } & \\
\hline $.1 . .1$ &.$/ M T$ & $. / M-1 / N Y$ & .180 & شريك & & \\
\hline
\end{tabular}

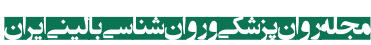

است كه تنظيم هيجان را در بستر روابط زناشويى مورد ارزيابى

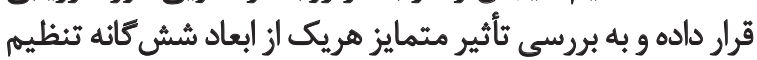

همسران و همجنين رابطه مؤلفه هاى تنظيم هيجان با رضايت

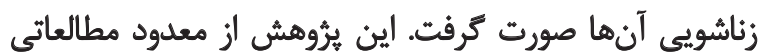


هيجانيذيرى منفى مردان بيشبينى إنينده ميزان رضايت

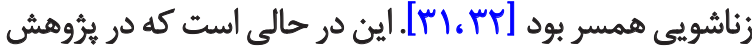

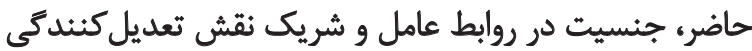

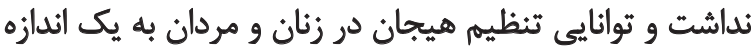

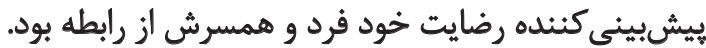
باور عمومى اين است كه در حوزههاى مختلف ياسخدهى

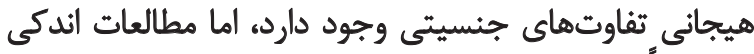

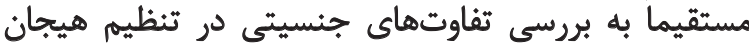

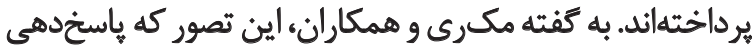

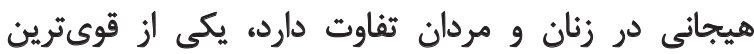

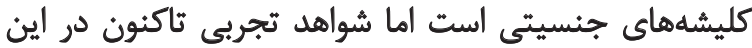

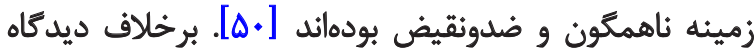

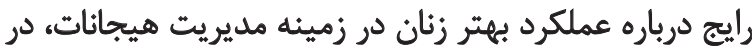

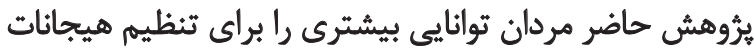

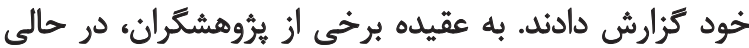

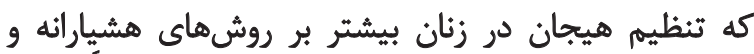

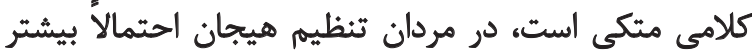

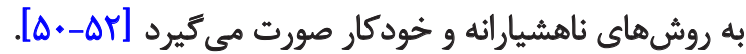
به عبارت ديكر، زنان و مردان در تنظيم عامدانه و آكاهانه هيجانات خود با يكديكر تفاوت ندارن ندارند، بلكه هنئكام تنام تنظيم

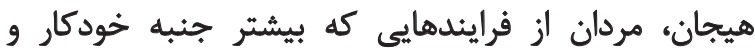

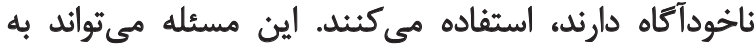

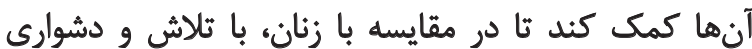

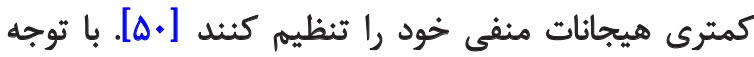

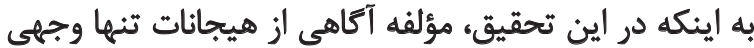

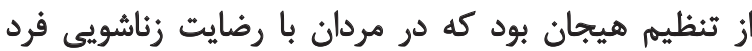

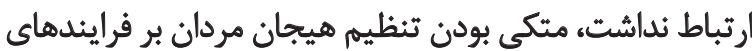

$$
\text { ناهشيار محتمل به نظر مىرسد. }
$$

همانطور كه فرض شده بود، علاوه بر نمره كلى تنظيم

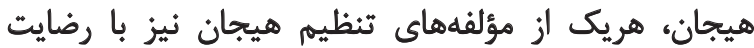

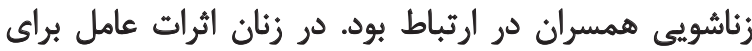

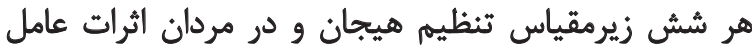

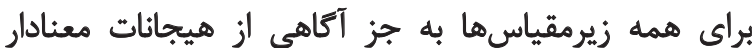

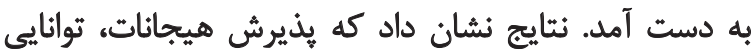

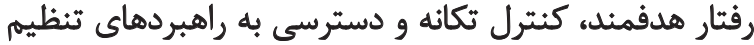

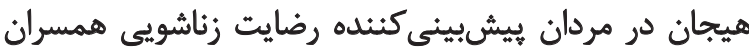

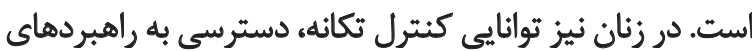

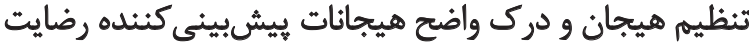

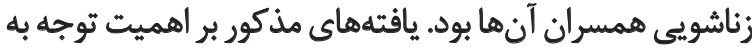

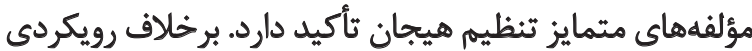

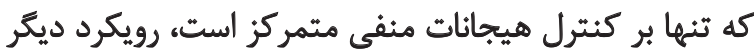

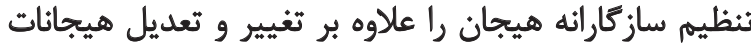

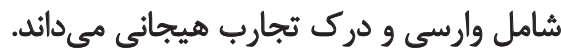

هيجان برداخته است. فرضيه اوليه اين مطالعهاء تنظيم هيجان

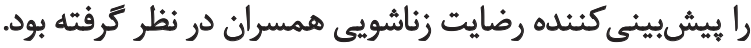

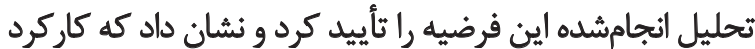

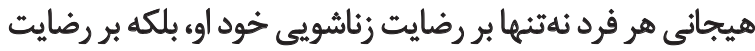

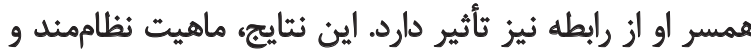

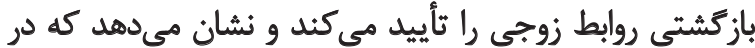

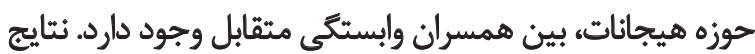

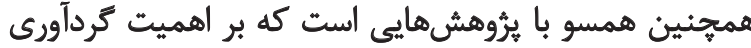

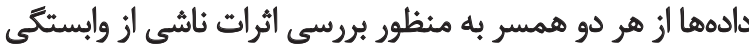

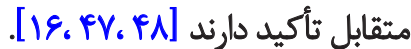
در حالى كه اثرات عامل از كذشته محل تمركز يُروهشَّران

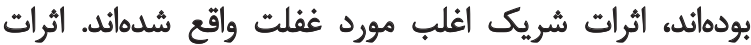

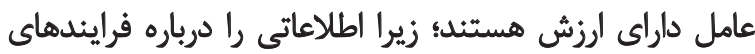

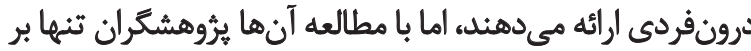

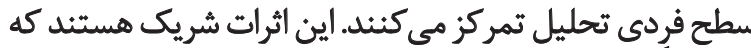

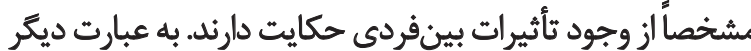

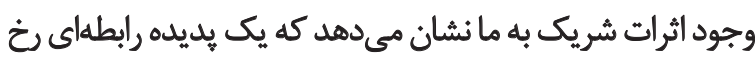

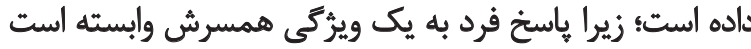

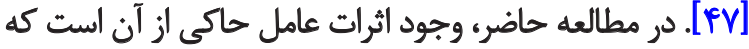

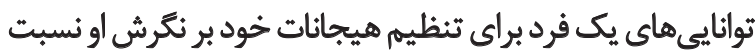

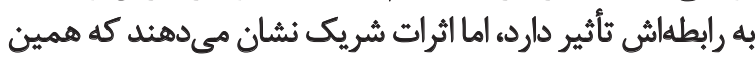

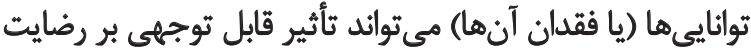

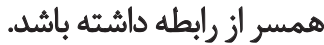

تعدادى از تحقيقات كذشته شواهدى را در دأئيد رابطه بين تنظيم هيجان و رضايت زناشويى ارائه دادهاند. در ئيك

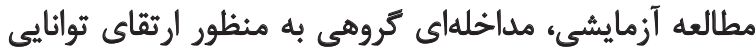

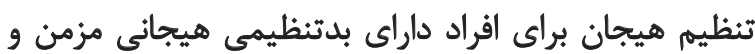

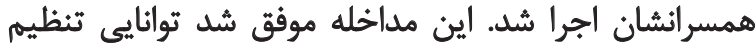

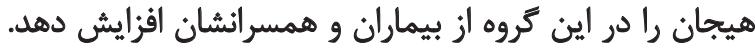

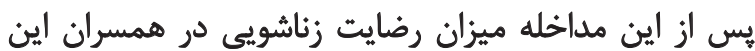

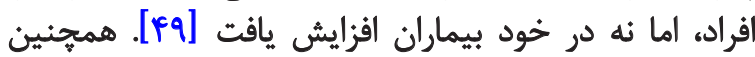

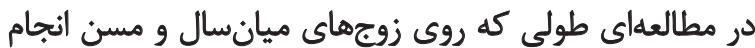

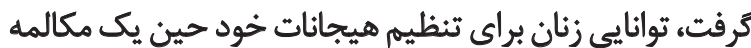

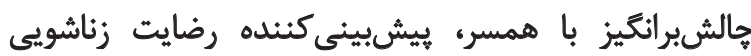

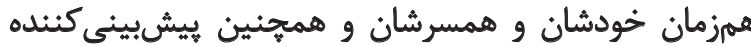
رضايت زناشويى همسرشان در طول زمان ونمان بود.

بين توانايى تنظيم هيجان مردان و رضايت زناشويى هيجيك

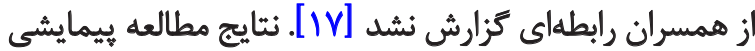

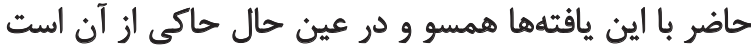

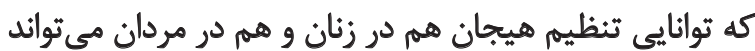

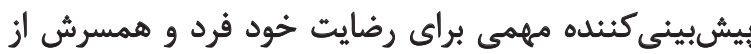

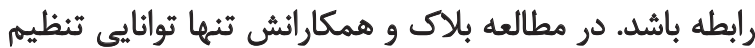

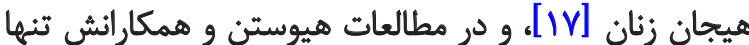


هيجانى همسران و متعاقباً حفظ كيفيت رابطه در نظر كرفت

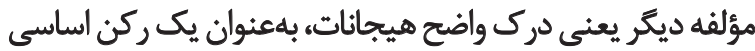

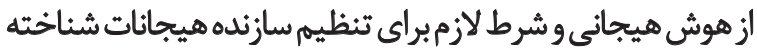

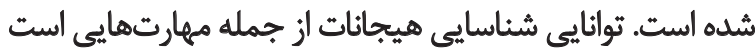

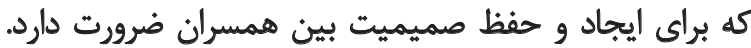

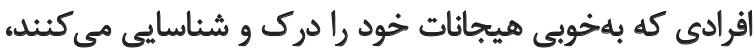

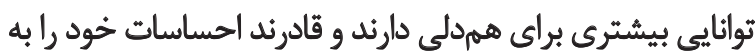

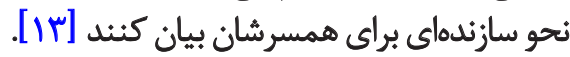

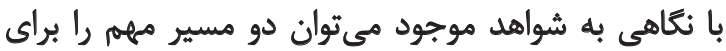

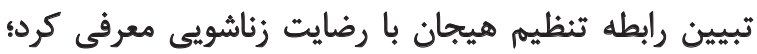

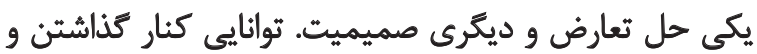

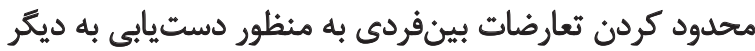

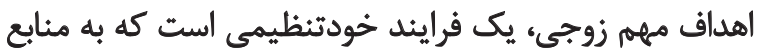

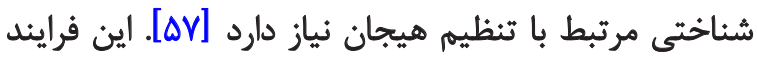

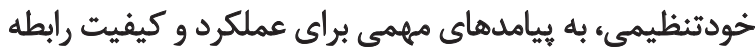

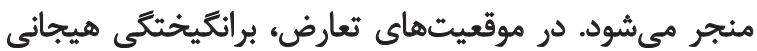

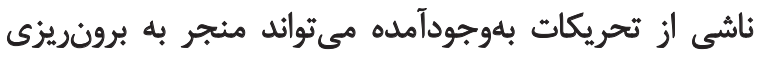

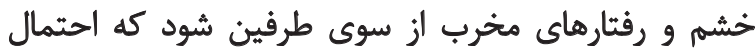

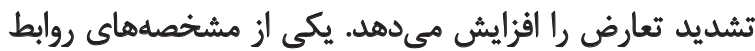

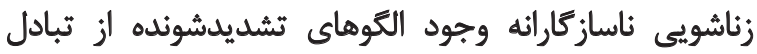

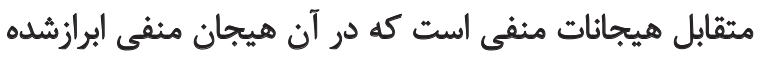

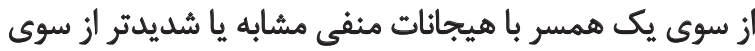

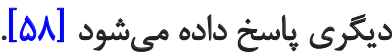

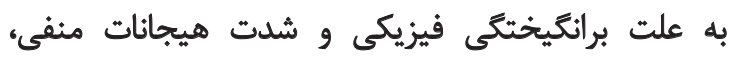

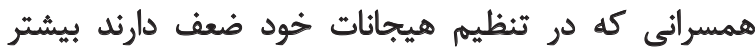

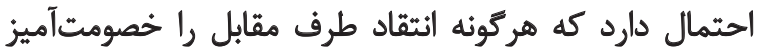

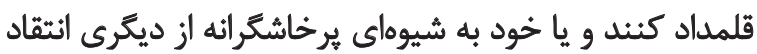

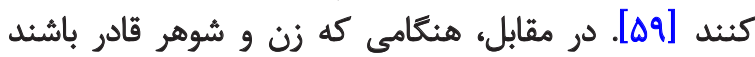

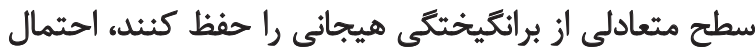

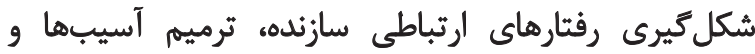

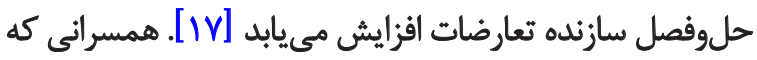

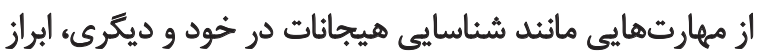

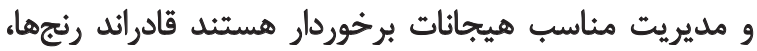

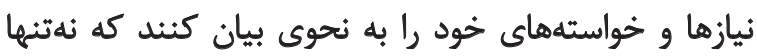

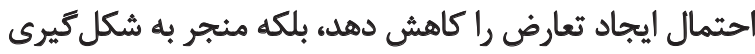

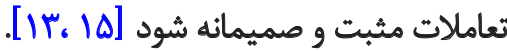

بسيارى از درمانهاى روانشناختى نوين بر مشكلات مرتبط

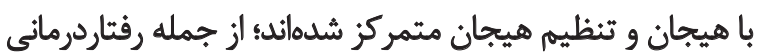

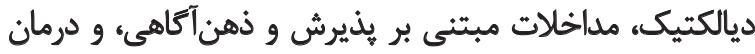

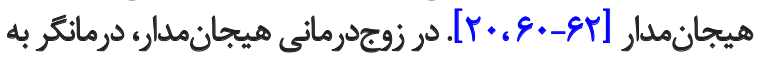

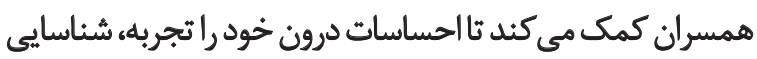

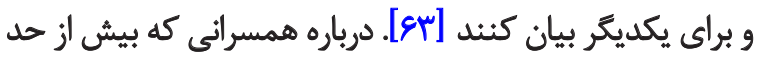

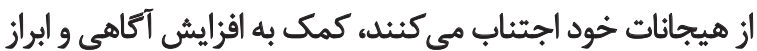

شواهدى مبنى بر تأثير هؤلفهاهاي تنظيم هيجان بر كيفيت

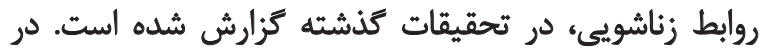

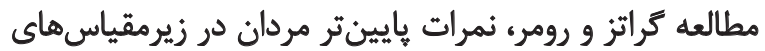

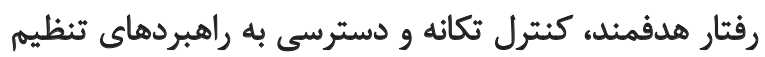

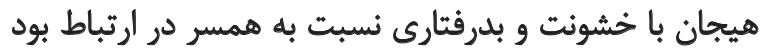

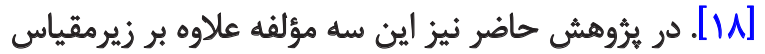

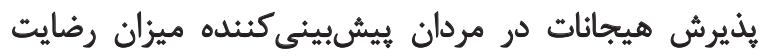

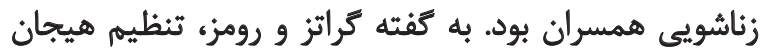

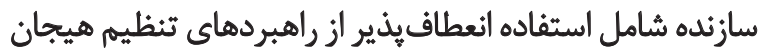

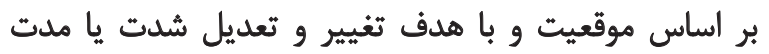

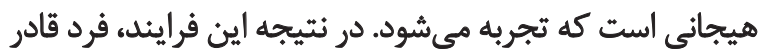

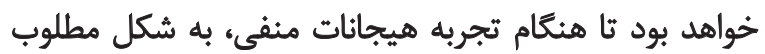

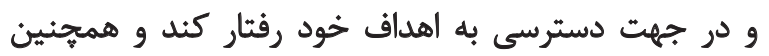

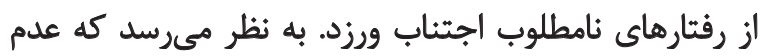

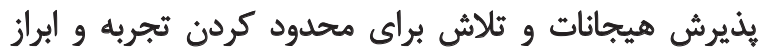

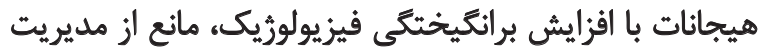

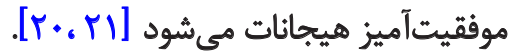
تحقيقات حاكى از آن است كه ناتوانى در كنترل تكانه دئ دري

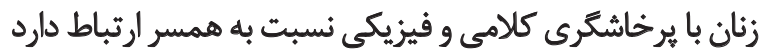

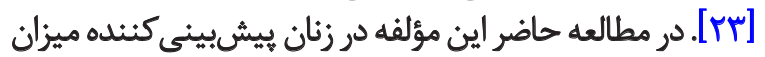

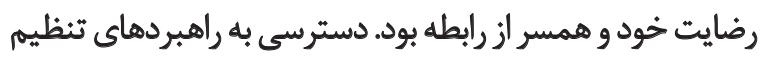

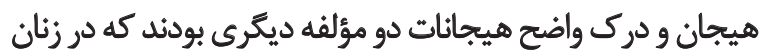

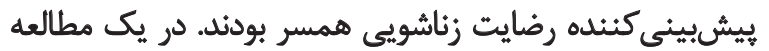

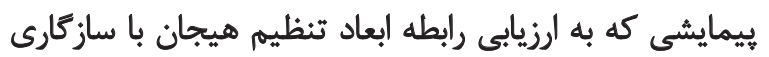

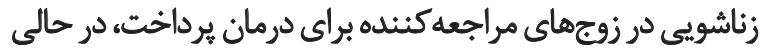

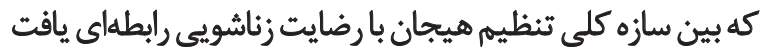

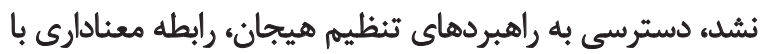

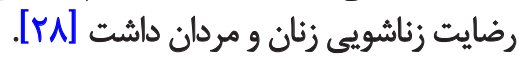
استفاده از راهبردهاى مختلف تنظيم هيجان مي تواند بيامدهاي

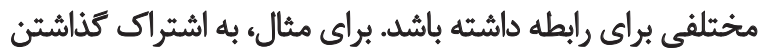

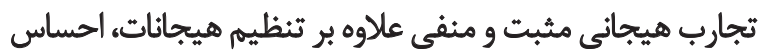

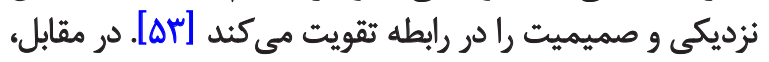

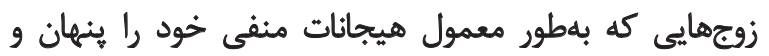

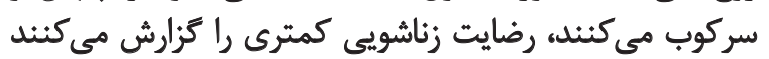

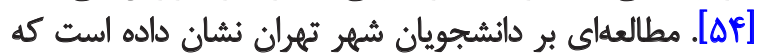

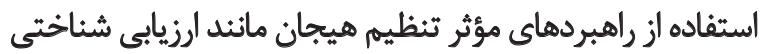

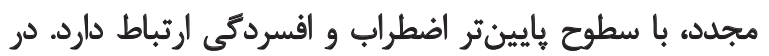

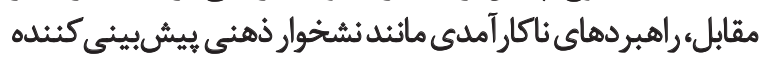

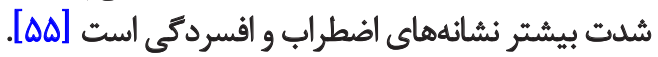

بادر نظر كرفتن شواهد تجربى متعددى كه حاكى از كاهش

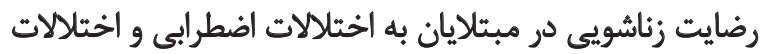

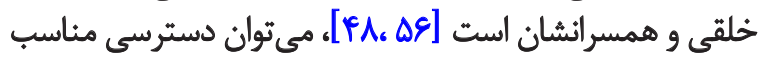

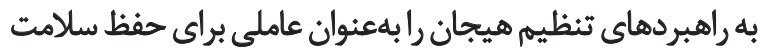


نهنتها از توانايى تنظيم هيجان خود آنهاء بلكه از توانايى تنظيم نئيم

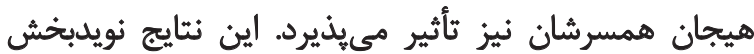

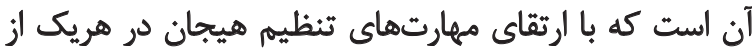

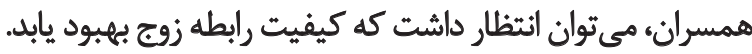

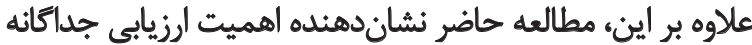

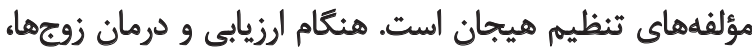

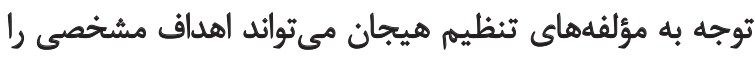

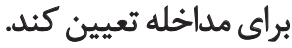

در زمينه نقش فرايندهاى تنظيم هيجان در روابط زوجى

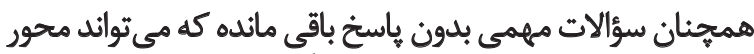

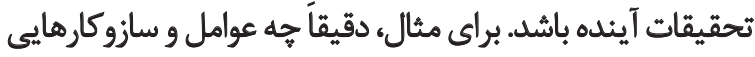

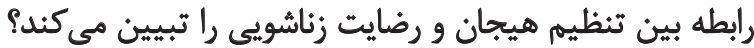

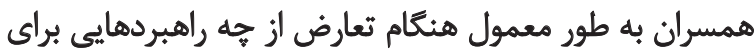

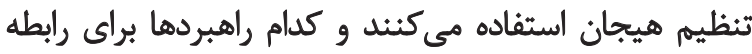

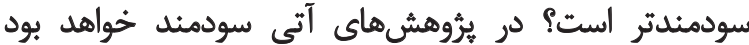

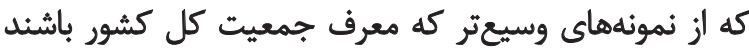

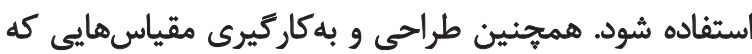

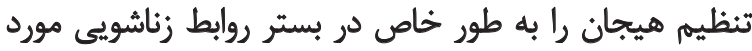

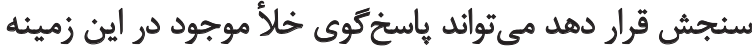

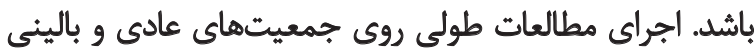

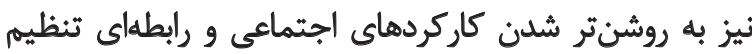

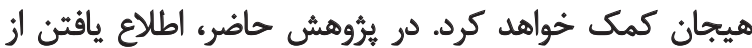

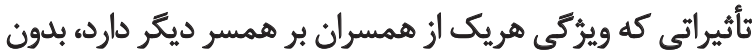

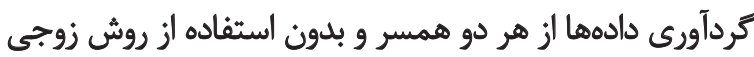

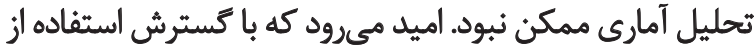

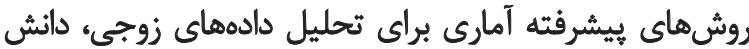

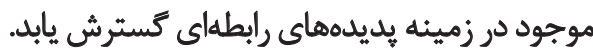

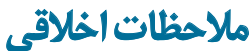

\section{ييروى از اصول اخلاق يُوهش}

اين تحقيق با رعايت اصول اخلاق برؤهش انجام شده است.

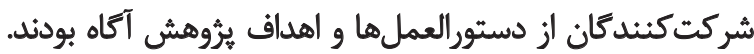

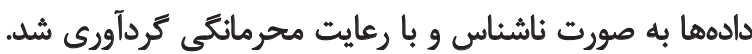

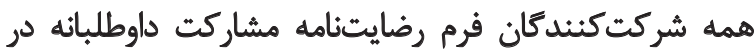

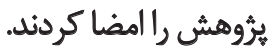

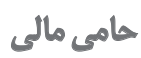

اين ئزوهش از هيج نهادى حمايت مالى دريافت نكرده و از ازئ

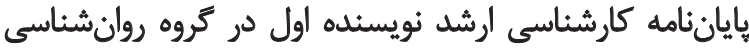

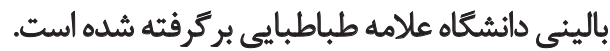

تجارب هيجانى مثبت و منفي از اهميت ويرُهاي برخوردار است.

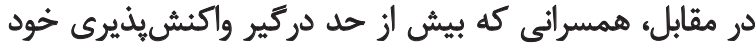

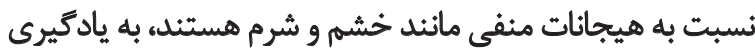

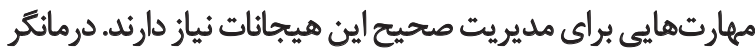

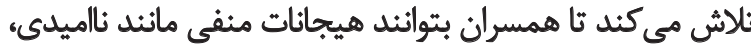

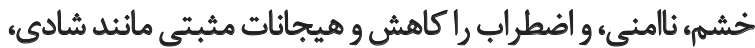

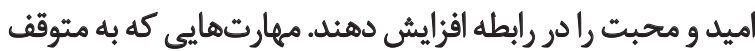

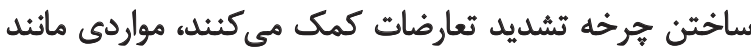

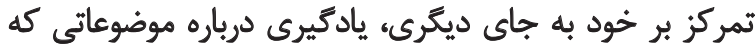

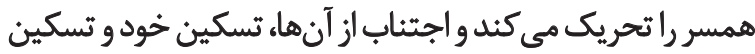

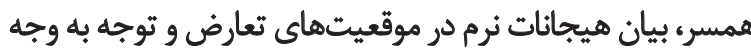

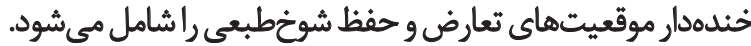
نتايج يروهش حاضر مىبايست بادر نظر داشتن محدوديتهاي

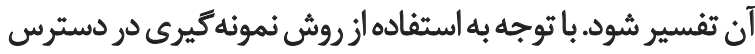

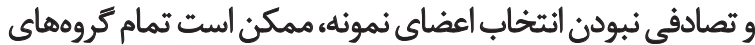

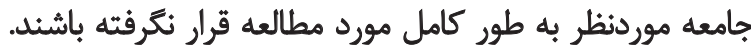

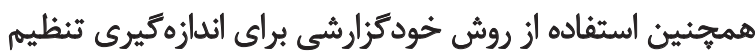

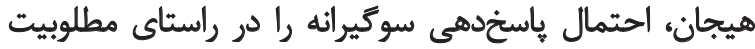

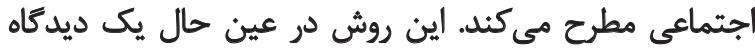

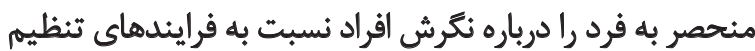

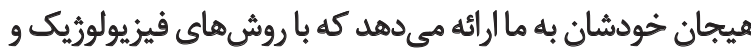

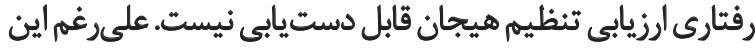

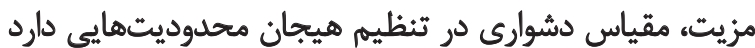

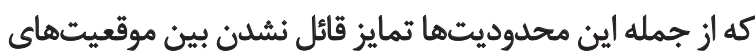

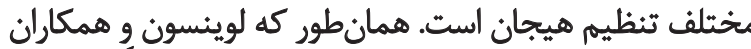

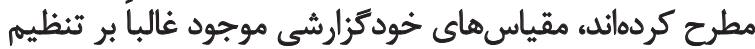

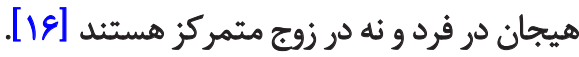
تمركز اين يرسشنامهها بر فرد باعث مىشود ابعادى از تنظيم

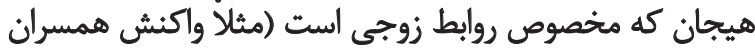

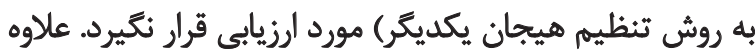

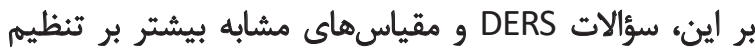

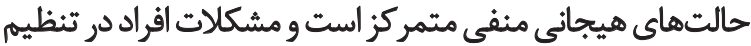

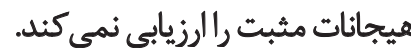

\section{نتيجليَيرى}

ازدواج بستر غنى و يويايى براى تجربه، ابراز و مديريت هيجانات

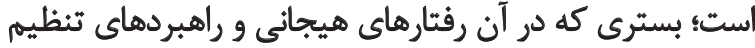

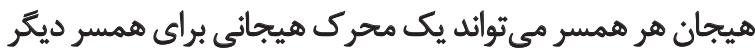

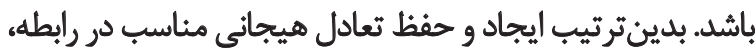

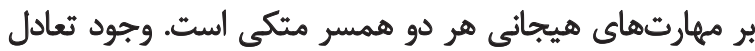

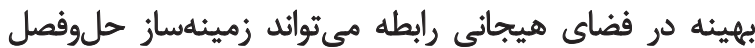

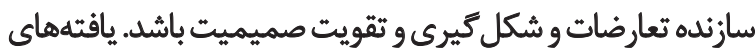

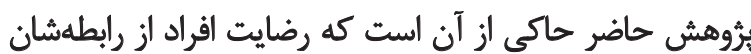




$$
\text { مشاركت نويسندكّان }
$$

مفهومسازي، اعتبارسنجى، مرور و ويرايش: همه نويسندكان؛

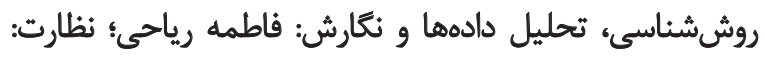

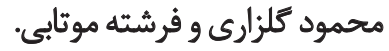

$$
\text { تعارض مناقع }
$$

بنابر اظهار نويسندكان، اين يُروهش تعارض منافع نداشته است.

$$
\text { تشكر و قدردانى }
$$

بدينوسيله از تمامى زوجهايى كه در يثوهش حاضر شركت

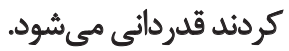




\section{References}

[1] Proulx CM, Helms HM, Buehler C. Marital quality and personal well-being: A meta-analysis. Journal of Marriage and Family. 2007; 69(3):576-93. [DOI:10.1111/j.17413737.2007.00393.x]

[2] Kiecolt-Glaser JK, Newton TL. Marriage and health: His and hers. Psychological Bulletin. 2001; 127(4):472-503. [DOI:10.1037/0033-2909.127.4.472] [PMID]

[3] Whisman MA. Marital distress and DSM-IV psychiatric disorders in a population-based national survey. Journal of Abnormal Psychology. 2007; 116(3):638-43. [DOI:10.1037/0021-843X.116.3.638] [PMID]

[4] Gottman JM, Levenson RW. Marital processes predictive of later dissolution: Behavior, physiology, and health. Journal of Personality and Social Psychology. 1992; 63(2):221-33. [DOI:10.1037/0022-3514.63.2.221]

[5] Bradbury TN, Fincham FD, Beach SRH. Research on the nature and determinants of marital satisfaction: A decade in review. Journal of Marriage and Family. 2000; 62(4):964-80. [DOI:10.1111/j.1741-3737.2000.00964.x]

[6] Rosen-Grandon JR, Myers JE, Hattie JA. The relationship between marital characteristics, marital interaction processes, and marital satisfaction. Journal of Counseling \& Development. 2004; 82(1):58-68. [DOI:10.1002/j.1556-6678.2004. tb00286.x]

[7] Iran's National Oraganization for Civil Registration. [Marriage and divorce data (Persian)] [Internet]. 2020 [Updated 2020 June 21]. Available from: https://www.sabteahval.ir/avej/Page.aspx?ID=3257\&Page=Magazines / SquareShowMagazine\&mId $=49826$

[8] Gross JJ. The emerging field of emotion regulation: An integrative review. Review of General Psychology. 1998; 2(3):271-99. [DOI:10.1037/1089-2680.2.3.271]

[9] Gross, JJ, Richards JM, John OP. Emotion regulation in everyday life. In: Snyder DK, Simpson J, Hughes JN, editors. Emotion Regulation in Couples and Families: Pathways to Dysfunction and Health. Washington, D.C. American Psychological Association; 2006. pp. 13-35. [DOI:10.1037/11468-001]

[10] Lopes PN, Nezlek JB, Extremera N, Hertel J, FernàndezBerrocal P, Schütz A, et al. Emotion regulation and the quality of social interaction: Does the ability to evaluate emotional situations and identify effective responses matter? Journal of Personality. 2011; 79(2):429-67. [DOI:10.1111/ j.1467-6494.2010.00689.x] [PMID]

[11] Eisenberg N, Fabes RA, Guthrie IK, Reiser M. Dispositional emotionality and regulation: Their role in predicting quality of social functioning. Journal of Personality and Social Psychology. 2000; 78(1):136-57. [DOI:10.1037/00223514.78.1.136]

[12] Lopes PN, Brackett MA, Nezlek JB, Schütz A, Sellin I, Salovey P. Emotional intelligence and social interaction. Personality and Social Psychology Bulletin. 2004; 30(8):1018-34. [DOI:10.1177/0146167204264762] [PMID]

[13] Cordova JV, Gee CB, Warren LZ. Emotional skillfulness in marriage: Intimacy as a mediator of the relationship be- tween emotional skillfulness and marital satisfaction. Journal of Social and Clinical Psychology. 2005; 24(2):218-35 [DOI:10.1521/jscp.24.2.218.62270]

[14] Mirgain SA, Cordova JV. Emotion skills and marital health: The association between observed and self-reported emotion skills, intimacy, and marital satisfaction. Journal of Social and Clinical Psychology. 2007; 26(9):983-1009. [DOI:10.1521/jscp.2007.26.9.983]

[15] Prager KJ. The dilemmas of intimacy: Conceptualization assessment, and treatment. New York: Routledge; 2014 [DOI:10.4324/9780203375372]

[16] Levenson RW, Haase CM, Bloch L, Holley SR, Seider BH. Emotion regulation in couples. In: Gross JJ, editor. HandBook of Emotion Regulation. New York: The Guilford Press; 2014. pp. 267-83. https://psycnet.apa.org/record/2013-44085-017

[17] Bloch L, Haase CM, Levenson RW. Emotion regulation predicts marital satisfaction: More than a wives' tale. Emotion. 2014; 14(1):130-44. [DOI:10.1037/a0034272] [PMID] [PMCID]

[18] Gratz KL, Roemer L. Multidimensional assessment of emotion regulation and dysregulation: Development, factor structure, and initial validation of the Difficulties in Emotion Regulation Scale. Journal of Psychopathology and Behavioral Assessment. 2004; 26(1):41-54. [DOI:10.1023/ B:JOBA.0000007455.08539.94]

[19] Rellini AH, Vujanovic AA, Gilbert M, Zvolensky MJ. Childhood maltreatment and difficulties in emotion regulation: Associations with sexual and relationship satisfaction among young adult women. The Journal of Sex Research. 2012; 49(5):434-42. [DOI:10.1080/00224499.2011.565430] [PMID]

[20] Hayes SC, Strosahl KD, Wilson KG. Acceptance and commitment therapy: An experiential approach to behavior change. New York: Guilford Publications; 1999. https:/ / books.google.com/books?id=ZCeB0JxG6EcC\&dq

[21] Paivio SC, Greenberg LS. Experiential theory of emotion applied to anxiety and depression. In: FlackWF, Laird JD, editors. Emotions in Psychopathology: Theory and Research. New York: Oxford University Press; 1998. pp. 229-42. https:/ / books.google.com/books?id=CPsmdnNESvEC\&dq

[22] Schafer J, Caetano R, Cunradi CB. A path model of risk factors for intimate partner violence among couples in the United States. Journal of Interpersonal Violence. 2004; 19(2):127-42. [DOI:10.1177/0886260503260244] [PMID]

[23] Shorey RC, Brasfield H, Febres J, Stuart GL. An examination of the association between difficulties with emotion regulation and dating violence perpetration. Journal of $\mathrm{Ag}$ gression, Maltreatment \& Trauma. 2011; 20(8):870-85. [DOI 10.1080/10926771.2011.629342] [PMID] [PMCID]

[24] Testa M, Leonard KE. The impact of marital aggression on women's psychological and marital functioning in a newlywed sample. Journal of Family Violence. 2001; 16(2):115-30. [DOI:10.1023/ A:1011154818394]

[25] Williams SL, Frieze IH. Patterns of violent relationships, psychological distress, and marital satisfaction in a national sample of men and women. Sex Roles. 2005; 52(11):771-84. [DOI:10.1007/s11199-005-4198-4] 
[26] Richards JM, Butler EA, Gross JJ. Emotion regulation in romantic relationships: The cognitive consequences of concealing feelings. Journal of Social and Personal Relationships. 2003; 20(5):599-620. [DOI:10.1177/02654075030205002]

[27] Shareh H, Eshaghi-Sani M. [Predictive role of morningness-eveningness personality, cognitive flexibility and cognitive emotion regulation in marital satisfaction in middleaged women (Persian)]. Iranian Journal of Psychiatry and Clinical Psychology. 2019; 24(4):384-99. [DOI:10.32598/ ijpcp.24.4.384]

[28] Rick JL, Falconier MK, Wittenborn AK. Emotion regulation dimensions and relationship satisfaction in clinical couples. Personal Relationships. 2017; 24(4):790-803. [DOI:10.1111/pere.12213]

[29] Zaki J, Williams WC. Interpersonal emotion regulation. Emotion. 2013; 13(5):803-10. [DOI:10.1037/a0033839] [PMID]

[30] Ben-Naim S, Hirschberger G, Ein-Dor T, Mikulincer M. An experimental study of emotion regulation during relationship conflict interactions: The moderating role of attachment orientations. Emotion. 2013; 13(3):506-19. [DOI:10.1037/a0031473] [PMID]

[31] Huston TL, Chorost AF. Behavioral buffers on the effects of negativity on marital satisfaction: A longitudinal study. Personal Relationships. 1994; 1(3):223-39. [DOI:10.1111/j.1475-6811.1994.tb00063.x]

[32] Huston TL, Vangelisti AL. Socioemotional behavior and satisfaction in marital relationships: A longitudinal study. Journal of Personality and Social Psychology. 1991; 61(5):721-33. [DOI:10.1037/0022-3514.61.5.721]

[33] APIMPower Program. Power analysis for the actor-partner interdependence model [Internet]. 2016 [Updated 2018 September 15]. Available from: https:/ / robert-a-ackerman. shinyapps.io/apimpower

[34] Spanier GB. Measuring dyadic adjustment: New scales for assessing the quality of marriage and similar dyads. Journal of Marriage and Family. 1976; 38(1):15-28. [DOI:10.2307/350547]

[35] Lambert JE. Adult attachment and romantic relationship quality: The role of emotional skillfulness [MA. thesis]. Albany, NY: State University of New York; 2008.

[36] Hassanshahi M, Noori A, Molavi H. [Relationship between locus of control and marital adjustment in secondary school teachers in Shiraz marital (Persian)]. Psychological Research. 2004; 7(1):51-61. https:/ / www.magiran.com/paper/199454

[37] Darvizeh Z, Kahaki F. [Relationship between marital adjustment and psychological well-being (Persian)]. Womens Studies. 2008; 6(1):91-104. https://www.sid.ir/fa/journal/ ViewPaper.aspx? $\mathrm{ID}=84083$

[38] Barazandeh H, Sahebi A, Aminyazdi A, Mahram B. [The relationship between marital adjustment and relationship standards (Persian)]. Journal of Developmental Psychology (Iranian Psychologists). 2006; 2(8):319-30. http://jip.azad. ac.ir/article_512418_en.html

[39] Mollazadeh J, Mansour M, Ezhehei J, Kiamanesh A. [Coping styles and marital adjustment among martyrs' children
(Persian)]. Journal of Psychology. 2002; 6(3):255-75. https:/ / www.sid.ir/fa/journal/ViewPaper.aspx?ID=27151

[40] Staples AM, Mohlman J. Psychometric properties of the GAD-Q-IV and DERS in older, community-dwelling GAD patients and controls. Journal of Anxiety Disorders. 2012; 26(3):385-92. [DOI:10.1016/j.janxdis.2012.01.005] [PMID]

[41] Weinberg A, Klonsky ED. Measurement of emotion dysregulation in adolescents. Psychological Assessment. 2009; 21(4):616-21. [DOI:10.1037/a0016669] [PMID]

[42] Khanzadeh M, Saeediyan M, Hosseinchari M, Edrissi F. [Factor structure and psychometric properties of difficulties in emotional regulation scale (Persian)]. International Journal of Behavioral Sciences. 2012; 6(1):87-96. http://www. behavsci.ir/article_67768.html

[43] Asgari P, Pasha GR, Aminiyan M. [Relationship between emotion regulation, mental stresses and body image with eating disorders of women (Persian)]. Andisheh va Raftar. 2009; 4(13):65-78. https://www.sid.ir/fa/journal/ViewPaper.aspx?ID $=127377$

[44] Alavi Kh. [The effectiveness of group dialectical behavior therapy in decreasing the symptoms of depression among Mashhad students (Persian)] [MSc. thesis]. Mashhad: Ferdowsi University of Mashhad; 2009. https:/ / ganj-old.irandoc.ac.ir/articles/497186

[45] Cook WL, Kenny DA. The actor-partner interdependence model: A model of bidirectional effects in developmental studies. International Journal of Behavioral Development. 2005; 29(2):101-9. [DOI:10.1080/01650250444000405]

[46] Kenny DA, Cook W. Partner effects in relationship research: Conceptual issues, analytic difficulties, and illustrations. Personal Relationships. 1999; 6(4):433-48. [DOI:10.1111/j.1475-6811.1999.tb00202.x]

[47] Kenny DA, Kashy DA, Cook WL. Dyadic data analysis. New York: Guilford Press; 2006. https://books.google. com/books?id=D7OyERQVQ1cC\&dq

[48] Whisman MA, Uebelacker LA, Weinstock LM. Psychopathology and marital satisfaction: The importance of evaluating both partners. Journal of Consulting and Clinical Psychology. 2004; 72(5):830-8. [DOI:10.1037/0022006X.72.5.830] [PMID]

[49] Kirby JS, Baucom DH. Treating emotion dysregulation in a couples context: A pilot study of a couples skills group intervention. Journal of Marital and Family Therapy. 2007; 33(3):375-91. [DOI:10.1111/j.1752-0606.2007.00037.x] [PMID]

[50] McRae K, Ochsner KN, Mauss IB, Gabrieli JJD, Gross JJ. Gender differences in emotion regulation: An fMRI study of cognitive reappraisal. Group Processes \& Intergroup Relations. 2008; 11(2):143-62. [DOI:10.1177/1368430207088035] [PMID] [PMCID]

[51] Barrett LF, Lane RD, Sechrest L, Schwartz GE. Sex differences in emotional awareness. Personality and Social Psychology Bulletin. 2000; 26(9):1027-35. [DOI:10.1177/01461672002611001]

[52] Nolen-Hoeksema S, Morrow J, Fredrickson BL. Response styles and the duration of episodes of depressed mood. Journal of Abnormal Psychology. 1993; 102(1):20-8. [DOI:10.1037/0021-843X.102.1.20] [PMID] 
[53] Rimé B. Interpersonal emotion regulation. In: Gross JJ, editor. Handbook of Emotion Regulation. New York: The Guilford Press; 2007. pp. 466-85. https://psycnet.apa.org/ record/2007-01392-023

[54] Feeney JA. Adult attachment, emotional control, and marital satisfaction. Personal Relationships. 1999; 6(2):16985. [DOI:10.1111/j.1475-6811.1999.tb00185.x]

[55] Ahmadian Vargahan F, Gharraee B, Atef Vahid MK, Habibi $\mathrm{M}$. [The role of perfectionism dimensions and emotion regulation strategies in predicting the severity of depressive and anxiety symptoms of university students (Persian)]. Iranian Journal of Psychiatry and Clinical Psychology. 2014; 20(2): 153-61. http:/ /ijpcp.iums.ac.ir/article-1-2205-en.html

[56] Benazon NR, Coyne JC. Living with a depressed spouse. Journal of Family Psychology. 2000; 14(1):71-9. [DOI:10.1037/0893-3200.14.1.71]

[57] Gross JJ, Thompson RA. Emotion regulation: Conceptual foundations. In: Gross JJ, editor. Handbook of Emotion Regulation. New York: The Guilford Press; 2007. pp. 3-24. https:/ / psycnet.apa.org/record/2007-01392-001

[58] Gottman JM, Levenson RW. Assessing the role of emotion in marriage. Behavioral Assessment. 1986; 8(1):31-48. https:/ / psycnet.apa.org/record/1987-00943-001

[59] Klein SR, Renshaw KD, Curby TW. Emotion regulation and perceptions of hostile and constructive criticism in romantic relationships. Behavior Therapy. 2016; 47(2):143-54 [DOI:10.1016/j.beth.2015.10.007] [PMID]

[60] Linehan M. Cognitive-behavioral treatment of borderline personality disorder. New York:TheGuilford Press; 1993. https:// books.google.com/books?id=UZim3OAPwe8C\&dq

[61] Segal ZV, Williams JMG, Teasdale JD. Mindfulnessbased cognitive therapy for depression: A new approach to preventing relapse. New York: Guilford Publications; 2002. https://books.google.com/books?id=_QJ3cQT5UPsC\&dq

[62] Greenberg LS. Emotion focused therapy: Coaching clients to work through their feelings. Washington, D.C.: American Psychological Association; 2002. [DOI:10.1037/10447-000]

[63] Goldman RN, Greenberg LS. Promoting emotional expression and emotion regulation in couples. In: Snyder DK, Simpson JA, Hughes JN, editors. Emotion Regulation in Couples and Families: Pathways to Dysfunction and Health. Washington, D.C.: American Psychological Association; 2006. pp. 231-48. [DOI:10.1037/11468-011] 
This Page Intentionally Left Blank 